\title{
Interaction Between Visual and Vestibular Signals for the Control of Rapid Eye Movements
}

\author{
A. D. VAN BEUZEKOM AND J.A.M. VAN GISBERGEN \\ Department of Biophysics, University of Nijmegen, $6500 \mathrm{HB}$ Nijmegen, The Netherlands
}

Received 2 November 2001; accepted in final form 25 February 2002

Van Beuzekom, A. D. and J.A.M. Van Gisbergen. Interaction between visual and vestibular signals for the control of rapid eye movements. J Neurophysiol 88: 306-322, 2002; 10.1152/jn.00902.2001. To investigate interactions between voluntary and reflexive eye movements, five subjects were asked to make pro- or anti-saccades to various oblique locations cued by a head-fixed flash while being rotated sinusoidally in yaw $(0.17 \mathrm{~Hz} ; 73 \%$ s peak velocity) in complete darkness. Eye movements were recorded with the coil technique. In the pro-saccade task, targeting responses showed clear compensation for the intervening nystagmus, but there was a marked increase in horizontal scatter. Most quick phases directed into the hemifield opposite to the flash (away trials) were suppressed from $\sim 100 \mathrm{~ms}$ onward. By contrast, quick phases directed into the hemifield of the flash (toward trials) continued virtually unabated until visually triggered saccades began to appear. From $80 \mathrm{~ms}$ onward, these vestibularly triggered movements showed signs of metrical modification by the visual signal. In the anti-saccade experiments, suppression of quick phases away from the flash was just as strong as in the pro-saccade experiments, and error rates in these trials were almost as low as in stationary control conditions. Suppression of quick phases directed toward the flash was a new phenomenon that emerged only in antisaccade experiments. Since this inhibition had a late onset and was only partial, error rates in anti-saccade toward trials were very high. At short latencies, both components of most rapid eye movements were wrongly directed toward the flash. This was followed by a stage with frequent incongruent responses in which unsuppressed quick phases provoked an incorrect horizontal movement, whereas the vertical component showed a correct anti-saccade response. At still longer latencies, most responses were correct in both components. The visual modification of shortlatency responses in both tasks showed that rapid eye movements could not simply be classified as either voluntary or reflexive, but suggested that signals underlying each class could merge into a compromise response. That vestibular rotation during the anti-saccade task may cause a wrongly directed horizontal component resembling a quick phase, combined with a vertical component expressing a correct anti-saccade signal, reveals a remarkable independence at the component level. These observations suggest that voluntary and involuntary movements can be programmed in parallel. This behavior is explained most parsimoniously by assuming that the two signals converge at a component-coding stage of the system, rather than at a vectorial coding stage.

\section{N T R O D U C T I O N}

At the level of the brain stem, the generation of voluntary saccades and of the reflexive quick phases of nystagmus relies on a shared neural network, involving burst cells and omnipause neurons, known as the pulse generator (for reviews see, Hepp et al. 1989; Keller 1991; Moschovakis et al. 1996; Scudder et al. 2002). This arrangement raises interesting ques-

Address for reprint requests: A. D. Van Beuzekom, 231 Dept. of Biophysics, University of Nijmegen, P.O. Box 9101, 6500 HB Nijmegen, The Netherlands. tions on how these systems interact when both pathways are activated simultaneously. Since the generation of saccades and quick phases has commonly been studied in isolation, the problem of how these systems can function in conjunction has largely been ignored.

It is known from neurophysiological studies that the vestibular driving signals, including the shared pulse generator for fast eye movements, are coded temporally at the component level. Excitatory burst cells specialized for horizontal and for vertical rapid eye movements have been identified in the pontine reticular formation and the rostral midbrain, respectively. These cells are recruited both during voluntary saccades and reflexive quick phases into their ON-direction (Keller 1974).

It is well-established that the pathways for saccades to visual, auditory, and tactile targets have already converged at the level of the superior colliculus (SC), which plays an important role in the sensory-motor transformation for the control of saccadic eye movements (Groh and Sparks 1996; Jay and Sparks 1987; Sparks 1986). Collicular neurons are organized into a two-dimensional topographic map, representing the contralateral hemifield, that specifies the relation between the locus of activity in the map and the saccade vector (Robinson 1972). In this way, goal-directed saccades are initially represented as vectors in spatially organized motor maps that are subsequently decomposed and transformed into the temporal code of the pulse generator. Interestingly, there is some evidence for quick-phase related activity in the collicular motor map (Schiller and Stryker 1972; Wurtz and Goldberg 1972). A systematic movement-field study, however, has never been undertaken so that virtually nothing is known on how the spatial distribution of this activity relates to the layout of the collicular map. The evidence for convergence both in the SC and at the level of the pulse generator implies that visual and vestibular signals for the generation of rapid eye movements may interact at two levels of coding (vectors and components).

In an earlier study (Van Beuzekom and Van Gisbergen 2002), we investigated the interaction in the monkey by eliciting saccades by microstimulation in the superior colliculus during passive head rotation. We found robust metric and kinematic effects that were predominant in the component aligned with rotation. The component-specific nature of the observed changes suggested that the effects may have been caused by convergence of saccadic and vestibular signals at a component-coding stage downstream of the colliculus.

The costs of publication of this article were defrayed in part by the payment of page charges. The article must therefore be hereby marked "advertisement" in accordance with 18 U.S.C. Section 1734 solely to indicate this fact. 
To investigate saccade-vestibular interactions under more natural conditions, saccades in the present study were elicited by presenting a flashed, head-fixed target at an oblique meridian while the subject was being rotated in yaw. Our main objective was to investigate how the saccadic system would cope with the interfering effects of ongoing horizontal nystagmus, quick phases in particular. We wondered whether there would be indications of a hierarchy allowing the saccadic targeting system to suppress the quick-phase system and investigated the possibility that the voluntary and the reflexive system would produce compromise responses. The results actually show signs of both scenarios.

Quick phases directed to the hemifield away from the flash were suppressed at short latencies, before voluntary saccades could be generated. By contrast, quick phases directed toward the flash were not suppressed and showed visual modification in their metrics at even shorter latencies. In an anti-saccade task, such unsuppressed quick phases led to frequent errors. In a substantial number of trials, we saw incongruent responses where the vertical component showed a correct anti-saccade response, whereas the horizontal component was wrongly directed toward the flash. We suggest that this behavior reflects convergence of parallel voluntary and reflexive signals, probably downstream from the colliculus. A preliminary report of our findings has appeared in abstract form (Van Gisbergen and Van Beuzekom 2000).

\section{METHODS}

\section{Subjects}

Five healthy male subjects, between 21 and 56 yr of age, participated in the experiments. Three of them $(A B, J G$, and $P M)$ had knowledge about the purpose of the experiments, whereas the other two were naive.

\section{Setup}

All experiments were conducted in a completely dark room. The subject was seated in a motor-driven vestibular stimulator that could be rotated about a vertical axis. Chair position was measured using a digital position encoder with an angular resolution of $0.04^{\circ}$. To avoid body movements, the subject's trunk was tightly fixed using adjustable shoulder and hip supports. The head was firmly stabilized in the natural upright position for looking straight-ahead with a padded adjustable helmet.

Two-dimensional monocular eye position was measured with the scleral search coil technique (Collewijn et al. 1975) using oscillating magnetic fields generated by two sets of orthogonal coils $(0.77 \times$ $0.77 \mathrm{~m}$ ) inside the vestibular stimulator. The signals from the eye coil were amplified, demodulated, and low-pass filtered $(200 \mathrm{~Hz})$ and sampled at $500 \mathrm{~Hz}$ per channel. Data were stored on hard disk for off-line analysis.

Visual targets were presented using an array of red light-emitting diodes (LEDs). LEDs were positioned on the intersections of five circles at $5,10, \ldots, 25^{\circ}$ and 12 meridians every $30^{\circ}$. The screen was attached to the vestibular stimulator with the center LED on the subject's nasooccipital axis at $0.39 \mathrm{~m}$ from the cyclopean eye. To calibrate the eye-coil signals, sessions started with a run in which subjects made refixations from the central fixation LED to each of 36 peripheral targets (eccentricity 10,20 , or $30^{\circ}$ ) and maintained fixation as long as it was visible.

\section{Paradigms}

Two paradigms, described in more detail below, were designed to collect oculomotor responses to flashed targets during vestibular rotation (visuo-vestibular paradigm) and to collect control data while the subject was stationary (visual paradigm). Prior to the calibration, subjects were given a practice run to get used to both paradigms. In all experiments, vision was binocular. Subjects never received feedback about their performance.

VISUAL STIMULATION. Two visual paradigms, involving a prosaccade and an anti-saccade task were used in separate sessions. In both paradigms, an LED was flashed for $4 \mathrm{~ms}$, at an eccentricity of $20^{\circ}$ in one of eight randomly chosen oblique directions [30, 60, 120, 150, $-30,-60,-120,-150^{\circ}$ ], where $0^{\circ}$ denotes rightward and $90^{\circ}$ points upward. In the pro-saccade task, the subject had to look straight-ahead until the target appeared and then shift gaze as accurately as possible to its remembered location. He was asked to recenter gaze after a short fixation period at target location. No central fixation LED was provided. Visual targets were presented at random times, with an intermediate time interval of at least $2 \mathrm{~s}$. Each run lasted $96 \mathrm{~s}$ and contained 32 target presentations, 4 times at each oblique meridian. In the anti-saccade task, the subject had to shift gaze toward the position opposite to the flashed target. Due to the more complex nature of this task, the time between 2 target presentations was increased so that 24 targets were flashed in every 96-s run.

VISUOVESTIBULAR STIMULATION. To test how targeting saccades would interact with vestibularly induced eye movements, the visual stimulation was combined with vestibular stimulation in the visuovestibular experiment. The subject, again instructed to look straight ahead, was rotated sinusoidally about the vertical axis at $0.17 \mathrm{~Hz}$ with a peak velocity of $73 \%$ s. Each run consisted of 16 sine periods. All subjects were tested using eight visuo-vestibular runs that were alternated with eight visual stimulation runs, described above. The instruction was identical in both conditions, but in the case of the visuovestibular experiments it was emphasized that the flashes were to be targeted in a head-centered frame of reference, moving with the rotating chair, rather than in an earth-fixed coordinate system.

\section{Data analysis}

Horizontal and vertical eye-coil signals were calibrated off-line using the fixation data obtained in the eye-coil calibration run (see above). Two neural networks, one for each eye-position component, were trained to fit the raw fixation data to the target locations (Melis and Van Gisbergen 1996). Each network consisted of two input units (representing the raw horizontal and vertical signal), three hidden units, and one output unit (representing the desired calibrated horizontal or vertical position signal). Raw eye-coil signals were subsequently calibrated by applying the resulting feed-forward networks. Calibration errors were typically $<0.5^{\circ}$, on average.

Detection of rapid eye movements, without making a distinction between saccades and quick phases, was performed on the calibrated eye position signals on the basis of separate velocity and acceleration/ deceleration criteria for onset and offset, respectively. Detection markings were manually adjusted, if necessary.

MARKING REFIXATION ENDPOINTS. In the pro-saccade task, targetdirected refixations usually consisted of multiple fast and slow movement contributions that brought the eyes from their initial position to the remembered flash location (see Fig. 1). To determine how vestibular stimulation affected targeting accuracy, it was necessary to determine which saccade could be regarded as the end of the refixation in response to a target flash. Since these final targeting responses could be most easily discerned in the vertical eye position channel, where the nystagmus had negligible effect, their identification was based on vertical eye position. Refixation offset was taken as the offset of the fast eye movement that brought the eyes to their most 

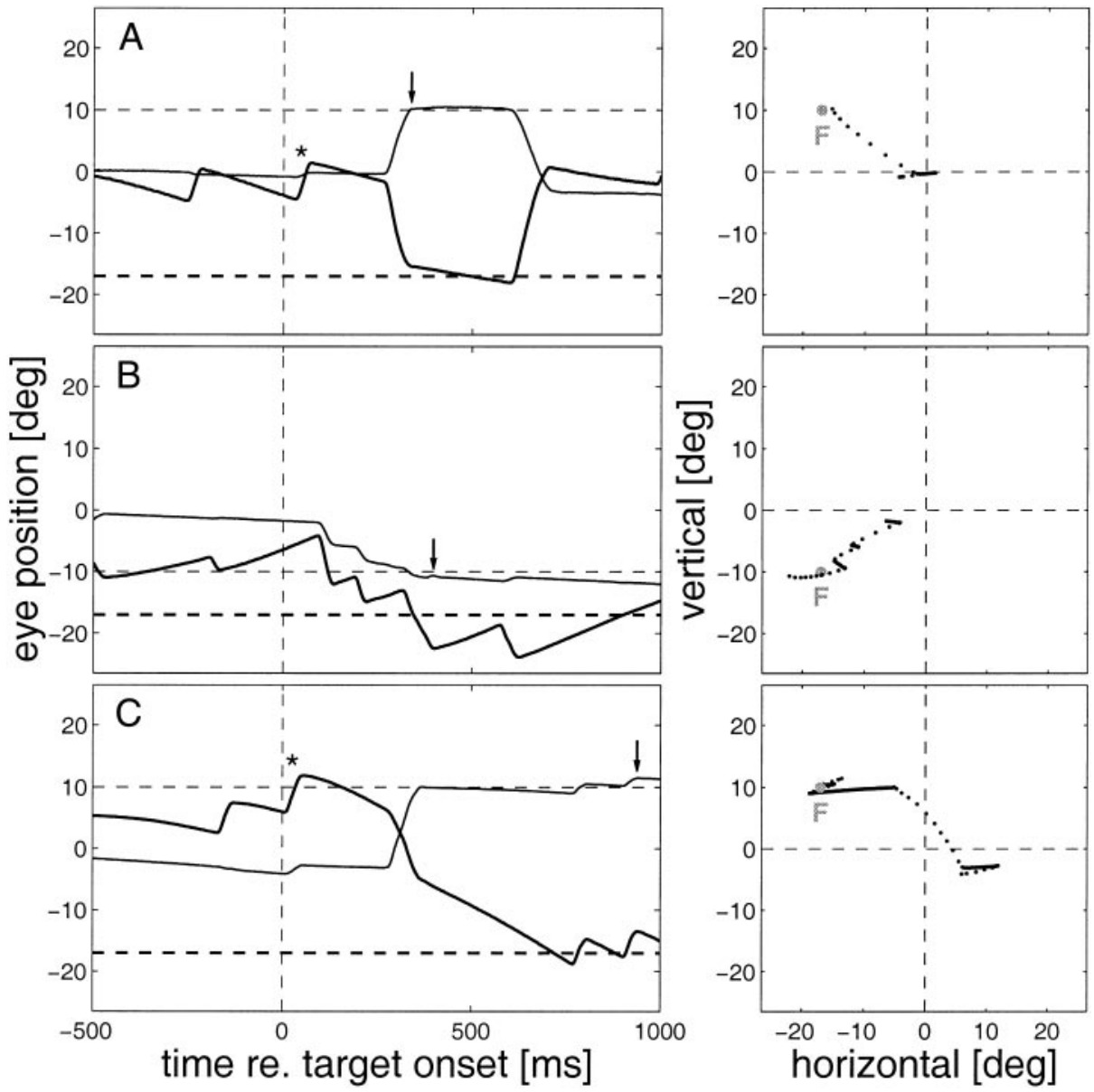

FIG. 1. Three examples of pro-saccade responses toward flashed targets during yaw rotation. The left-hand column shows horizontal (thick trace) and vertical (thin trace) eye position traces as a function of time. The vertical dashed line marks the time of the flash, the horizontal dashed lines denote target position. The arrow indicates refixation offset. Quick phases that occurred just after the target was presented have been marked with an asterisk (*). The corresponding spatial trajectories from target onset until refixation offset are shown in the right-hand column. The small gray disk labeled F denotes flash location. Data from subjects $A B$ and $J G$. extreme point of vertical excursion in response to the flash. Refixation offset was often marked by a corrective saccade with a vertical component, sometimes preceded by other corrective saccades. To exclude pure quick phases, which resumed as the eye reached the target area, fast eye movements with vertical displacements below $1^{\circ}$ were rejected as refixation endpoints. The result of this procedure was displayed graphically for each trial and corrected if necessary. Trials that failed to show any convincing saccadic response to the flash were excluded from further analysis $(\sim 1.5 \%)$. Three examples of prosaccade responses to a flashed target are shown in Fig. 1, together with the refixation endpoints that have been marked by an arrow.

\section{R E S U L T S}

Oculomotor responses to flashed targets in oblique directions were recorded during ongoing horizontal vestibular nystagmus to investigate the interaction between voluntary and reflexive eye movements. By way of introduction, we start with some general observations on performance in the pro-saccade task.

\section{Characteristics of pro-saccade targeting responses: qualitative observations}

As explained before (see METHODS), the subject was rotated sinusoidally about the vertical axis in complete darkness. He was instructed to look straight ahead between trials and to redirect gaze as accurately as possible toward the head-centered location of the target flash as soon as it appeared. Figure 1 shows three examples of eye movement recordings in re- sponse to a head-fixed flash at the location indicated by the horizontal dashed lines, presented at time 0. Horizontal (thick trace) and vertical (thin trace) eye position have been plotted as a function of time in the left-hand panels. The right-hand panels provide a spatial representation of the trajectory of the eye movement from target onset until refixation offset (marked by an arrow in the left-hand panels, see METHODS for procedure). Although the gain of the vestibuloocular reflex (VOR) was only 0.30 on average across subjects (range 0.15-0.43), there was still a substantial amount of nystagmus both before and after refixations. As a result, targeting responses only shifted the zone where nystagmus occurred and did not end with a period of steady fixation. The low gain of the VOR is probably related to the instruction to perform the task in a head-centered reference frame and the requirement to look straight ahead when awaiting the next target (Barr et al. 1976).

For the purpose of further analysis, it appeared useful to classify trials based on the relation between the direction of quick phases at the time of target presentation and the position of the flash. Trials were termed "toward" when quick phases moved the eye toward the hemifield containing the flash and were denoted as "away" trials when the quick phases were into the opposite direction. It should be noticed that the terms "toward" and "away" refer to relationships in the horizontal channel. Since quick phases were about horizontal and flashes were presented on oblique meridians, precise vectorial alignment of quick phases and retinal error was extremely rare. For simplicity, trials were regarded as toward trials when the target 
was presented in the direction of chair rotation at the moment of the flash. Figure $1 B$ illustrates a toward trial; the other two examples show away trials.

The examples illustrate noteworthy differences among responses: first, while there are examples of rather accurate refixations (Fig. 1, $A$ and $C$ ), there is also a case with clear overshoot (Fig. 1B). Response accuracy will be discussed in more detail below. Moreover, it can be seen that a single rapid movement was sometimes sufficient to reach the target (Fig. $1 A)$, but other refixations consisted of several such responses (Fig. $1 B$ ). An interesting question, subject of extensive analysis later on, concerns the origin of these rapid eye movements: can they be classified as either of vestibular origin (quick phases) or visually mediated (saccades), or do intermediate types also exist? Some preliminary observations can be made. For example, the first rapid eye movements after the flash in examples $A$ and $C$ lack a clear goal-directed vertical component and therefore must have been pure quick phases $(*)$. It would be impossible to make such categorizing statements for every individual fast eye movement. However, as demonstrated below, it is possible to make such distinctions on a statistical basis. Before we address this issue further, we will concentrate on the question of how vestibular stimulation affected response accuracy.

\section{Effect of vestibular rotation on targeting accuracy in prosaccades}

Figure 2 shows eye position at the time of the flash $(\bigcirc)$ and the end positions of the final rapid eye movement (O) of all refixations made in one experimental session for two subjects. In the stationary condition (Fig. 2, $A$ and $C$ ), refixation endpoints showed clearly separated clusters associated with the eight different target locations. However, as the endpoint scatter and the presence of offsets indicate, responses were not flawless. The ellipses, which contain $95 \%$ of the refixation end positions for each target, are more or less of equal size. Note that the long axes of the ellipses are roughly oriented toward the straight ahead position, indicating that the variation in refixation amplitude is larger than the variability in refixation direction. Also notice that, due to the absence of a central fixation LED, initial eye positions showed considerable scatter.

Figure 2, $B$ and $D$, shows the refixation start and endpoint distributions during vestibular stimulation. It is immediately clear that the yaw rotation deteriorated performance in both subjects. Endpoint scatter is clearly larger, yielding bigger ellipses that sometimes even overlap. In contrast to the stationary condition, the long axes of the ellipses now tend to be horizontal, indicating that yaw rotation led to more scatter in the horizontal component. Yaw rotation also increased scatter in eye position at the moment of the flash $(O)$.

Figure 3 shows the final refixation-error distributions pooled from all subjects for each component. Positive errors reflect overshoots, whereas negative values indicate undershoots. In the stationary condition, errors in both horizontal (Fig. 3A) and vertical (Fig. $3 B$ ) component were modest and scattered around zero. As already illustrated in Fig. 2, rotation led to poorer performance (Fig. 3, $C$ and $D$ ). Refixation errors still scattered around zero, but covered an expanded range. This increase was

\section{stationary}
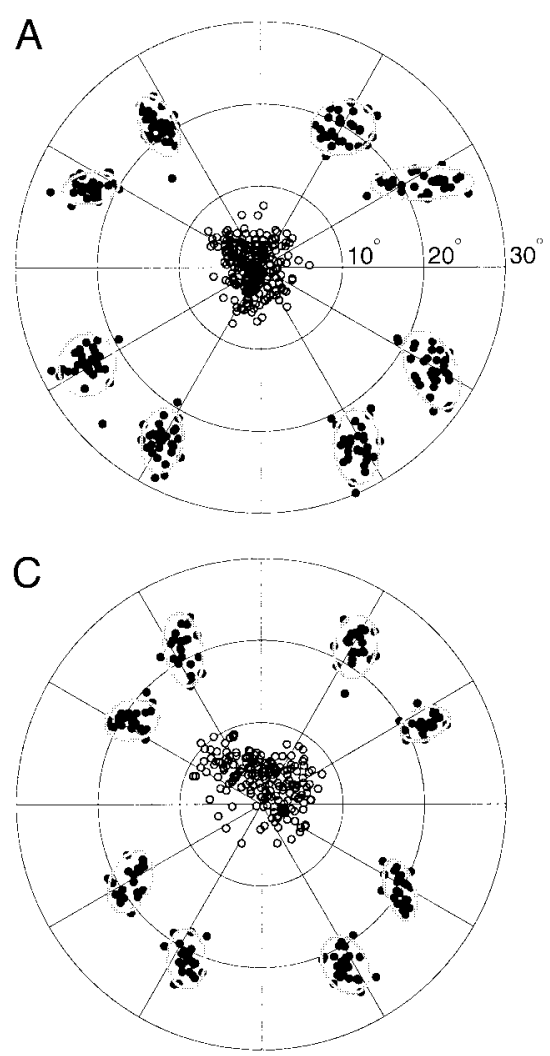

\section{yaw rotation}
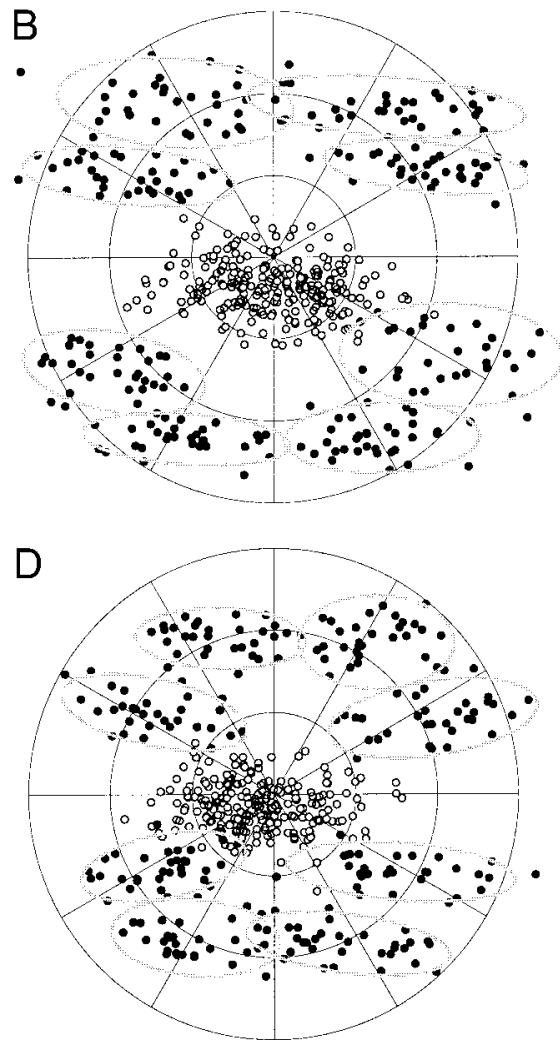

FIG. 2. Onset and offset positions of refixations in pro-saccade task. Eye positions at the time of the flash $(\bigcirc)$ and at the end of the refixation $(\bullet)$ toward a briefly flashed visual target are shown in head coordinates. Targets were presented at an eccentricity of $20^{\circ}$ relative to straight ahead, in eight oblique directions (see METHODS). The top panels present all refixation endpoints of subject $A B$, the bottom panels of subject $S P$. Refixations, that may be comprised of multiple saccades, ended near the target position in the stationary control condition (left-hand panels). End-point scatter was characterized using a principal components analysis (Sokal and Rohlf 1981). Using this procedure, 2 axes were determined: the principal axis corresponding to the direction of largest variability and the orthogonal axis. With these 2 axes an ellipse was constructed that contained $95 \%$ of the data points. During yaw rotation $(B$ and $D)$, responses were less accurate, as shown by the larger ellipses. Ellipse orientation also changed: yaw rotation mainly increased scatter in the horizontal component. 


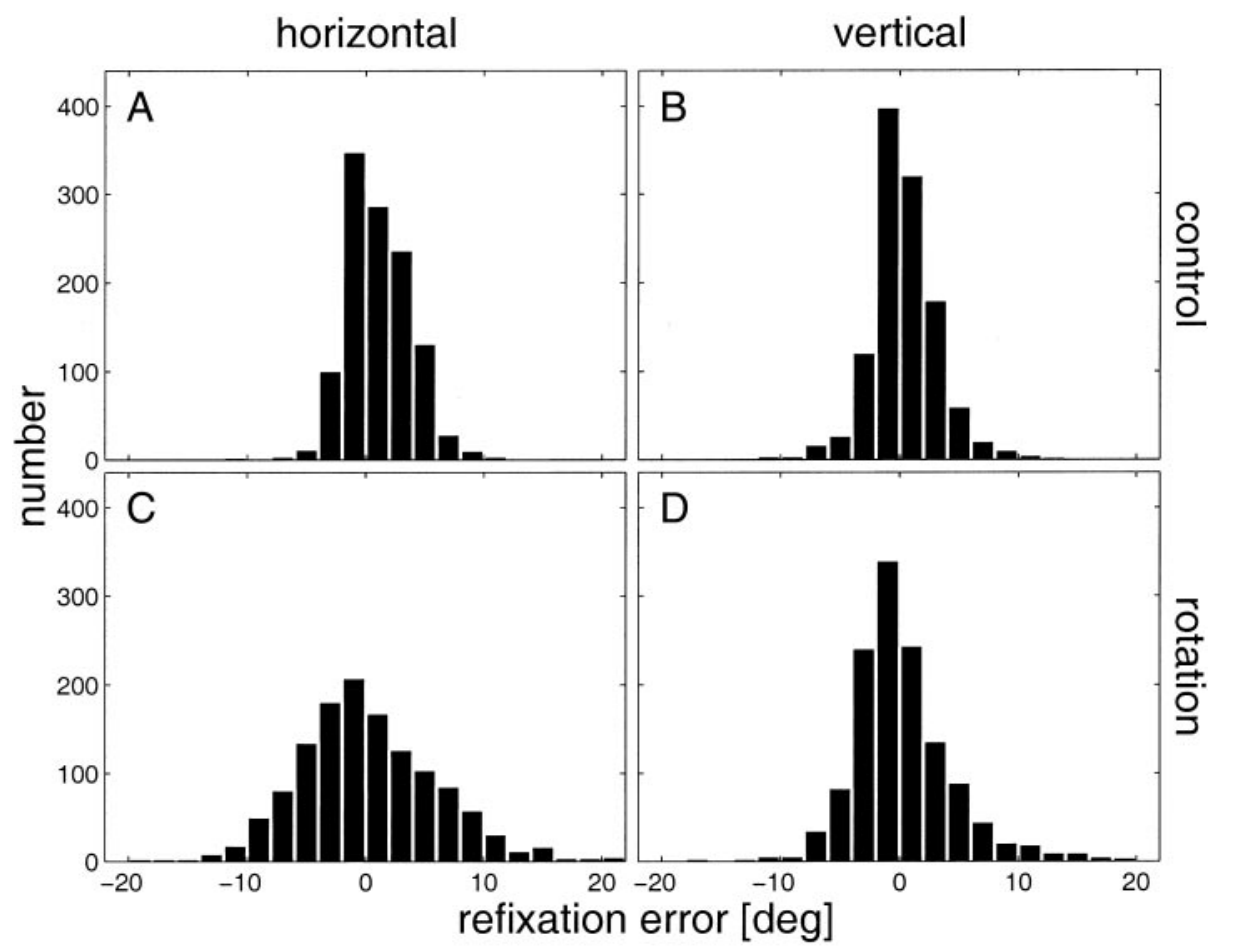

FIG. 3. Refixation error distributions in the pro-saccade task. Refixation errors (positive for overshoots, negative for undershoots) increased during vestibular stimulation, especially in the horizontal component. Pooled data from all subjects.

present in the vertical component, where the SD increased by $\sim 50 \%$, but was more pronounced in the horizontal component, where the SD was doubled. It should be noticed that rotation itself increases the difficulty in determining endpoints. Therefore the increased scatter could reside in part on the problems with defining the endpoint.

The examples in Fig. 1 clearly show that vestibular eye movements, slow phases and quick phases, were not suppressed after the presentation of the flash. Despite the low gain of the VOR (see above), slow phases in trials without intervening quick phases (e.g., Fig. $1 B$ ) caused an absolute cumulative horizontal displacement of $4.6 \pm 3.9^{\circ}$ (mean \pm SD). Trials where intervening quick phases occurred before the first saccade (as in Fig. 1, $A$ and $C$ ), yielded a net horizontal displacement of $4.2 \pm 3.9^{\circ}$. To make an accurate response, the oculomotor system had to take this movement into account. In the case of away trials (see Fig. 1, $A$ and $C$ ) the nystagmus may cause a net displacement of the eye toward the target. As a result, the required total saccadic displacement may be less than retinal error at the time of the flash. Nystagmic eye movements in toward trials may cause an opposite net shift (see Fig. $1 B$ ), and thus require a total saccadic displacement larger than retinal error. For each refixation response we computed the net horizontal vestibular eye displacement and the total horizontal saccadic displacement. Subsequently, both displacements were normalized. The normalized vestibular displacement was defined as the ratio of the total horizontal displacement due to vestibular eye movements (sum of all slow and intervening quick phases) and horizontal retinal error at the time of the flash. Similarly, normalized saccadic displacement was taken as the ratio of horizontal saccadic displacement and horizontal retinal error. Accordingly, when the sum of the normalized net vestibular and saccadic displacements equals one, the refixation is accurate. Since the results might be different for trials containing one or more intervening quick phases before the first saccade (for example, Fig. 1, $A$ and $C$ ), compared with those with only slow phases (for example, Fig. $1 B)$, the analysis was performed for each trial category separately.

The top row in Fig. 4 shows the normalized vestibular and saccadic displacements in the refixation, pooled from all subjects, for away (Fig. 4A) and toward (Fig. 4B) trials without quick phases. The results for trials containing quick phases are shown in the bottom row, again for away (Fig. 4C) and toward trials (Fig. 4D) separately. If the saccadic system had made a response reflecting retinal error, without compensating for the intervening nystagmus, its normalized displacement would equal 1 for all trials. To be on target, the response should be on the thin line with slope -1 representing perfect compensation (vestibular + saccadic $=1$ ). In most away trials with only slow phases (top row), vestibular displacements were positive, indicating net eye movements toward the flash, necessitating smaller saccadic responses. By contrast, toward trials typically had negative vestibular displacements, requiring larger saccadic displacements. As the scatter plots show, there is a clear trend that the saccadic system, on average, took these variations in vestibular eye displacement into account. The scatter is considerable, but both fit lines are close to the ideal relation. As can be seen in the intercepts, there is a slight trend toward undershoot in the away trials and overshoot in the toward condition. Trials containing one or more quick phases (see bottom row) also showed a clear trend toward compensation, both in toward and away trials. However, the undershoot in away trials and overshoot in toward trials was significantly more pronounced. Overall, compensation was clearly poorer in trials containing quick phases.

In what follows we will concentrate on a further characterization of the rapid eye movements after the flash without focusing on their degree of accuracy. The purpose of this 


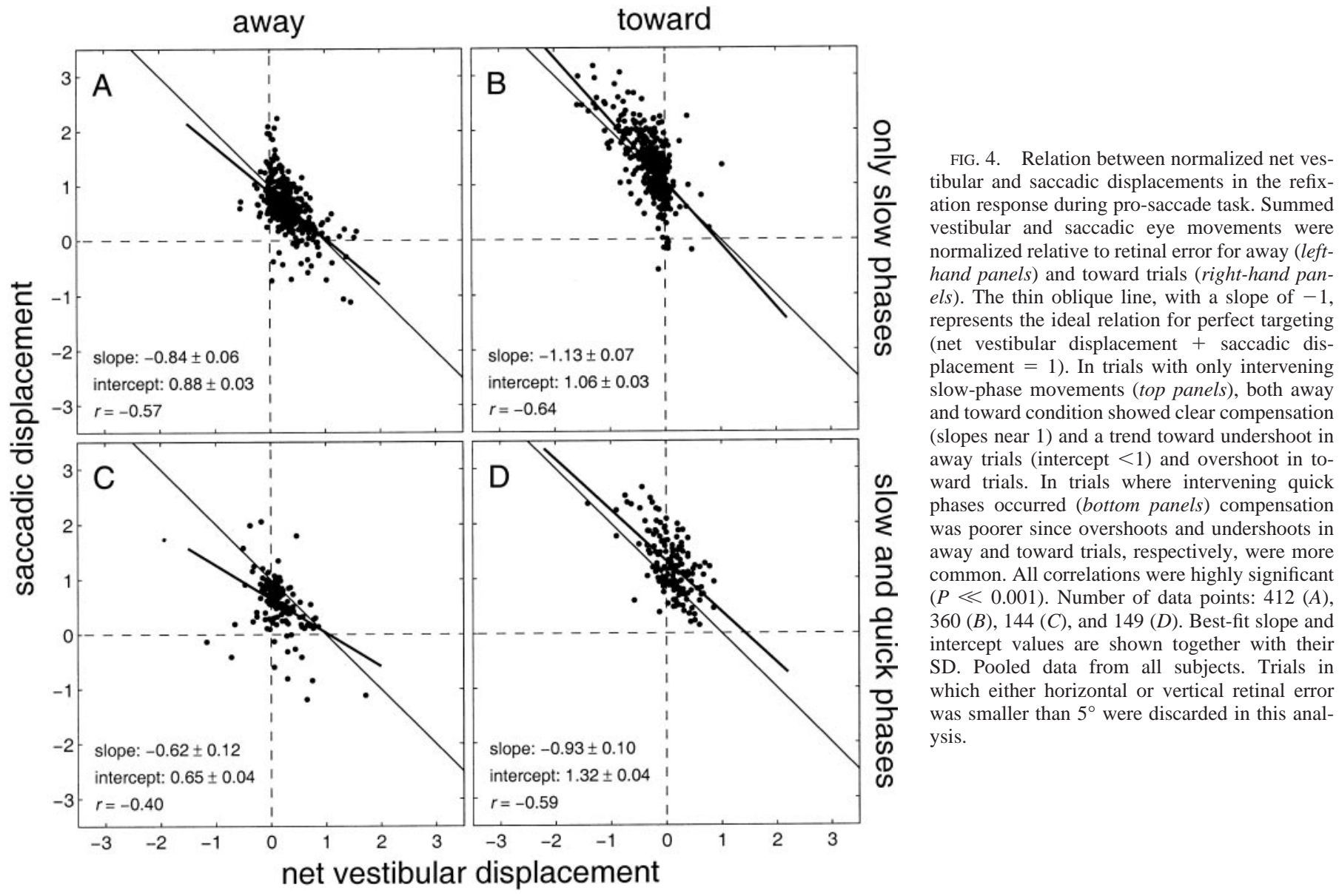

analysis is to describe the transition from pure quick phases immediately after the flash to goal-directed movements later on.

\section{Temporal stages of visual-vestibular interaction in pro- saccade experiments}

FIRST EFFECT OF FLASH ON METRICS: MODIFICATION LATENCY. As a first step in assessing the transition from pure quick phases to visually influenced movements, we compared the contribution of each rapid eye movement to the required refixation response imposed by the retinal location of the flash. The horizontal contribution of a given rapid eye movement was defined as the ratio of its horizontal displacement and the required horizontal refixation amplitude (i.e., horizontal retinal error). The vertical contribution was determined in a similar fashion. Defined in this way, eye movements with positive contributions are directed toward the flash, whereas a negative contribution indicates a wrongly directed eye movement, away from the flash. As shown in Fig. 5, rapid eye movements that preceded the flash (the latter marked by an arrow) were also normalized using the same definition to establish a baseline for pure quick phases. Since targets were presented in oblique directions, most refixations also required vertical contributions. By contrast, quick phases stood out by being nearly horizontal with their vertical contributions scattering around zero. Note that the corresponding horizontal contributions were positive during toward trials (Fig. 5A) and negative in away trials (Fig. $5 B)$. The sum of all horizontal contributions in a toward trial was usually larger than one, due to the intervening slow phase eye movements that moved the eye away from the target. In away trials, on the other hand, the sum could be less than one.

The scatter plots in Fig. 6 show the vertical (Fig. 6, $A$ and $B$ ) and horizontal (Fig. 6, $C$ and $D$ ) contributions of all rapid eye movements made by subject SP between $300 \mathrm{~ms}$ before and $800 \mathrm{~ms}$ after target presentation. Each data point represents a rapid eye movement. The bold lines depict median values of the data in a 40-ms time window that moved in steps of $5 \mathrm{~ms}$. We show median rather than mean values because this measure appears less sensitive to outliers. From before the target was presented until some time after the flash, we see a background level of rapid eye movements, most of them quick phases of nystagmus. Some are voluntary refixation movements made when the subject tried to maintain gaze at the straight-ahead position. As expected, the vertical contributions of these early eye movements scattered around zero (Fig. 6, $A$ and $B$ ). The first expression of a response to the visual target is observed in toward trials (right-hand column) where small positive vertical contributions occurred at short latencies, $75 \mathrm{~ms}$ in this subject. These small target-directed contributions, we suggest, probably stem from vestibularly initiated quick phases whose metrics were affected by the emerging visual target signals. To quantify the temporal onset of this phenomenon, vertical-modification latency was defined as the time when the running median first remained for at least $50 \mathrm{~ms}$ above its level of fluctuation in the $500 \mathrm{~ms}$ before the flash. The threshold criterion for detecting such a consistent deviation was based on the scatter 

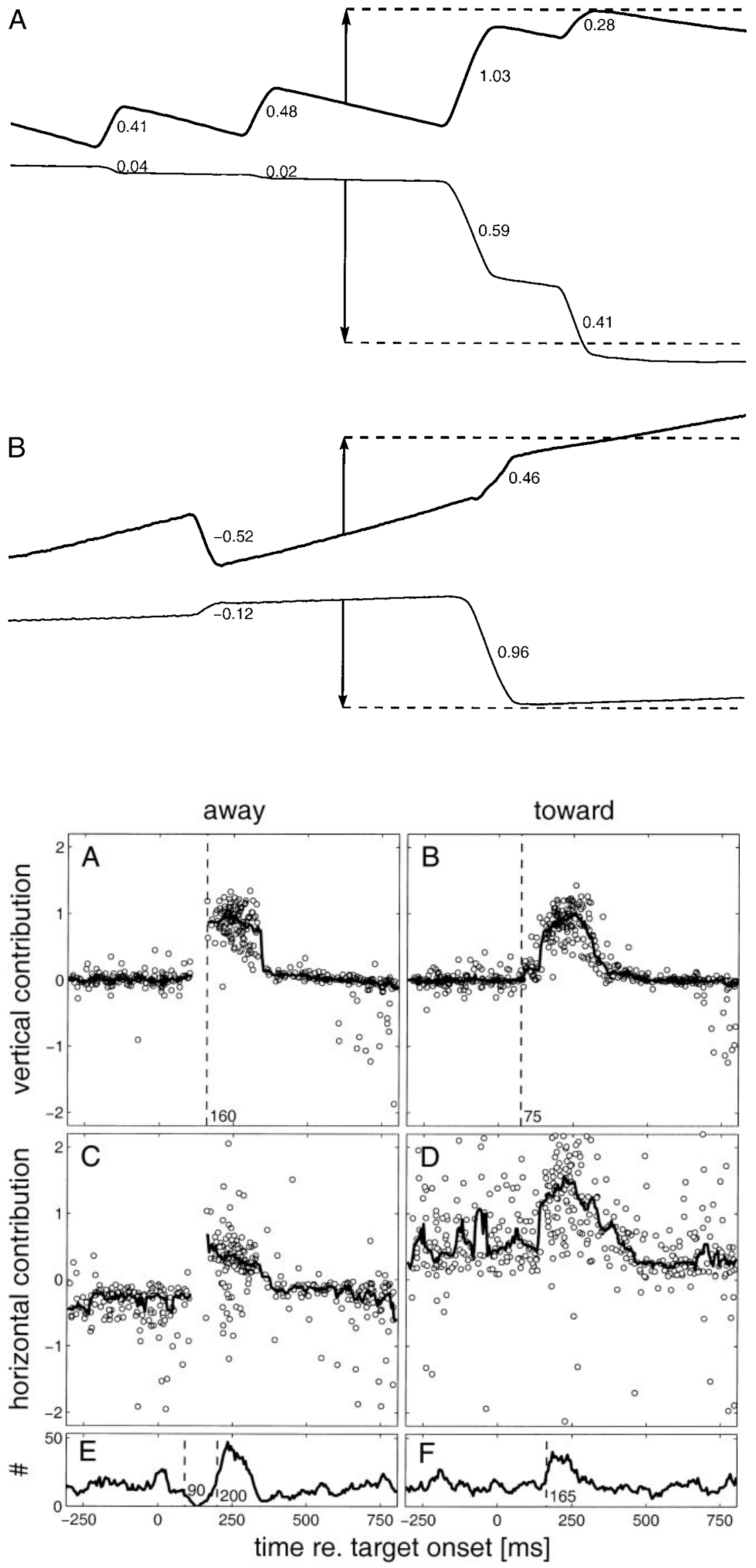

FIG. 5. Examples of rapid eye movement contributions to pro-saccade targeting response. The contribution of a rapid eye movement was defined as the ratio of its displacement and the required response amplitude at the time of the flash (i.e., retinal error in the pro-saccade task). Two examples of horizontal (thick trace) and vertical (thin trace) eye position traces are shown as a function of time. The vertical arrows mark the timing of the flash and point to the accompanying required end positions. Numbers show rapid eye movement contribution in each component. Note that in the toward example $(A)$, quick phases preceding the flash have a positive horizontal contribution, whereas away quick phases are characterized by negative contributions $(B)$.
FIG. 6. Signs of visual-vestibular interaction in the prosaccade task. The contributions of all rapid eye movements in the time window starting $300 \mathrm{~ms}$ before until $800 \mathrm{~ms}$ after target presentation are shown in $A$ and $B$ (vertical component) and $C$ and $D$ (horizontal component). Trials in which either horizontal or vertical retinal error was smaller than $5^{\circ}$ were discarded in this analysis. Bold lines are running medians in a 40-ms time window that moved along the time axis in steps of $5 \mathrm{~ms}$. $E$ and $F$ show the number of rapid eye movements in the same time window. The vertical contributions of the eye movements made before the flash, mostly quick phases, scatter around zero. In the toward condition (right-hand column), rapid eye movements start showing positive vertical contributions from approximately $75 \mathrm{~ms}$ after the flash onward, indicating that their vertical components were target directed. The corresponding horizontal components do not clearly exhibit this short-latency response (see, however, Fig. 7). In the away trials, rapid eye movement were suppressed after approximately $90 \mathrm{~ms}$ after target presentation $(E)$. Vertical dashed lines mark various latency parameters, see text for details. Data from subject $S P$. 
in running median values in the same preflash period and was set at two SDs above the mean. Such early modification emerged in the toward trials of all subjects. In away trials, most quick phases in this time window were suppressed (see following text for description).

Due to the ongoing nystagmus, horizontal contributions showed much more scatter and yielded a less clear picture. Away trials were characterized by negative contributions in the period preceding target onset, simply reflecting the fact that the quick phases in these trials moved the eyes away from the target (Fig. 6C). The opposite holds for the toward contributions in the same time window (Fig. $6 D$ ). Note that the horizontal contributions of quick phases preceding the flash show more scatter in toward trials compared with away trials. In toward trials, retinal error tends to be smaller due to the shift of the beating field (see, e.g., Chun and Robinson 1978) into the direction of the flash. Horizontal retinal error in pooled toward trials was $11.8 \pm 6.1^{\circ}$, compared with $15.4 \pm 6.2^{\circ}$ in pooled away trials (mean $\pm \mathrm{SD}$ ). Since we plot contributions (movement amplitude as a fraction of retinal error) this may cause large values when retinal error becomes small even when quick phase amplitude remains constant. Due to the relatively large scatter in contributions, assessment of the horizontal modification latency using the level discrimination was less reliable and was not attempted in data from individual subjects.

FLASH EFFECTS ON INITIATION OF RAPID EYE MOVEMENTS. To show flash effects on the initiation of rapid eye movements, Fig. $6, E$ and $F$, display their frequency as a smoothed function of time. As a clear sign that the visual stimulus caused quickphase suppression in away trials, we see a dip in the frequency plot starting $\sim 90 \mathrm{~ms}$ after the target flash. Such a period of rapid eye movement suppression was not seen in toward trials. Apparently, the presentation of the visual target only led to suppression of quick phases that would move the eye in a completely wrong direction. Suppression onset was determined as the time when the running median dropped two SDs below the mean median value for at least $30 \mathrm{~ms}$. It seems reasonable to suggest that the subsequent increase in the frequency of rapid eye movements represents visually triggered eye movements. Accordingly, we defined visual saccade latency as the time when the average exceeded the mean plus two SDs for at least $50 \mathrm{~ms}$. Notice that, although both modification latency and visual saccade latency reflect effects of the visual signal, there is a clear difference. The former represents the first effect of the visual stimulus on quick-phase metrics and is not ac- companied by any change in the initiation of rapid eye movements. The latter describes the onset of a significant increase in the frequency of rapid eye movements, associated with the first visually triggered eye movements.

The data in Fig. 6 indicate that the visual stimulus can modify the metrics of ongoing quick phases long before it can initiate goal-directed saccades by its own. We will now extend this comparison of various latency measures to all subjects.

COMPARISON OF VARIOUS LATENCY MEASURES. Table 1 summarizes all latency measures derived from the pro-saccade results. Two interesting features in the data of subject $S P$ (see Fig. 6) were typical for other subjects as well. First, all subjects showed modification of the vertical component in toward trials at very short latencies ( $80 \mathrm{~ms}$ on average). Second, suppression of rapid eye movements directed away from the flash started at slightly longer latencies (mean: $100 \mathrm{~ms}$ ) in four of five subjects. Thus both in toward and away trials, an effect of the visual stimulus could already be discerned at a time well before it started triggering voluntary saccades. Note that vertical modification latencies in the away trials always exceeded the suppression latencies. It remains uncertain whether early modification simply does not exist in away trials or whether quickphase suppression made it hard to detect.

The visual-saccade latency data (Table 1, right-hand columns) provided no evidence for temporal facilitation when quick phases and saccades were directed into the same hemifield. On average, the toward values were virtually identical to the values in the stationary control condition. By contrast, vestibular stimulation did have a clear effect on visual saccade latencies in away trials. In such conditions, which led to quick phases opposite to the required targeting saccade, visual latencies were clearly prolonged. One may wonder whether the occurrence of quick-phase suppression, which was a typical phenomenon in these trials but not in toward trials, may have played a role here. In other words, is it possible that the suppression mechanism has delayed the generation of visually triggered saccades? The data from subject PM, who lacked quick-phase suppression but had a clearly prolonged saccade latency anyway, argues against this hypothesis.

As stated earlier, data from individual subjects were too noisy to allow reliable estimates of modification latency in the horizontal component. We checked whether pooling the data could resolve this problem. Figure 7 presents the relative contributions of pooled data from all subjects except $P M$, who did not show quick-phase suppression. Note that the pooled

TABLE 1. Temporal characteristics of flash-induced metric and timing effects in the pro-saccade task

\begin{tabular}{|c|c|c|c|c|c|c|c|}
\hline Subject & Away & Toward & Away & Toward & Away & Toward & Control \\
\hline$J G$ & 145 & 80 & 95 & & 165 & 115 & 165 \\
\hline$M Z$ & 165 & 90 & 110 & & 215 & 185 & 155 \\
\hline$P M$ & 90 & 70 & & & 170 & 105 & 100 \\
\hline
\end{tabular}

Modification latency represents the time when the 1st visually induced metric changes became visible in the vertical component. In the toward condition, these latencies were very short, $80 \mathrm{~ms}$ on average. Note that the modification latency in the away trials always exceeded the suppression latency, indicating there actually was no early metric modification in these trials. Suppression latency marks the time when there was a decrease in the number of quick phases. Four of the 5 subjects showed this suppression in the away condition, with an average latency of $100 \mathrm{~ms}$. Only subject $A B$ showed suppression in the toward trials. Note that visual saccade latency, based on the number of rapid eye movements, was prolonged in the away condition. 


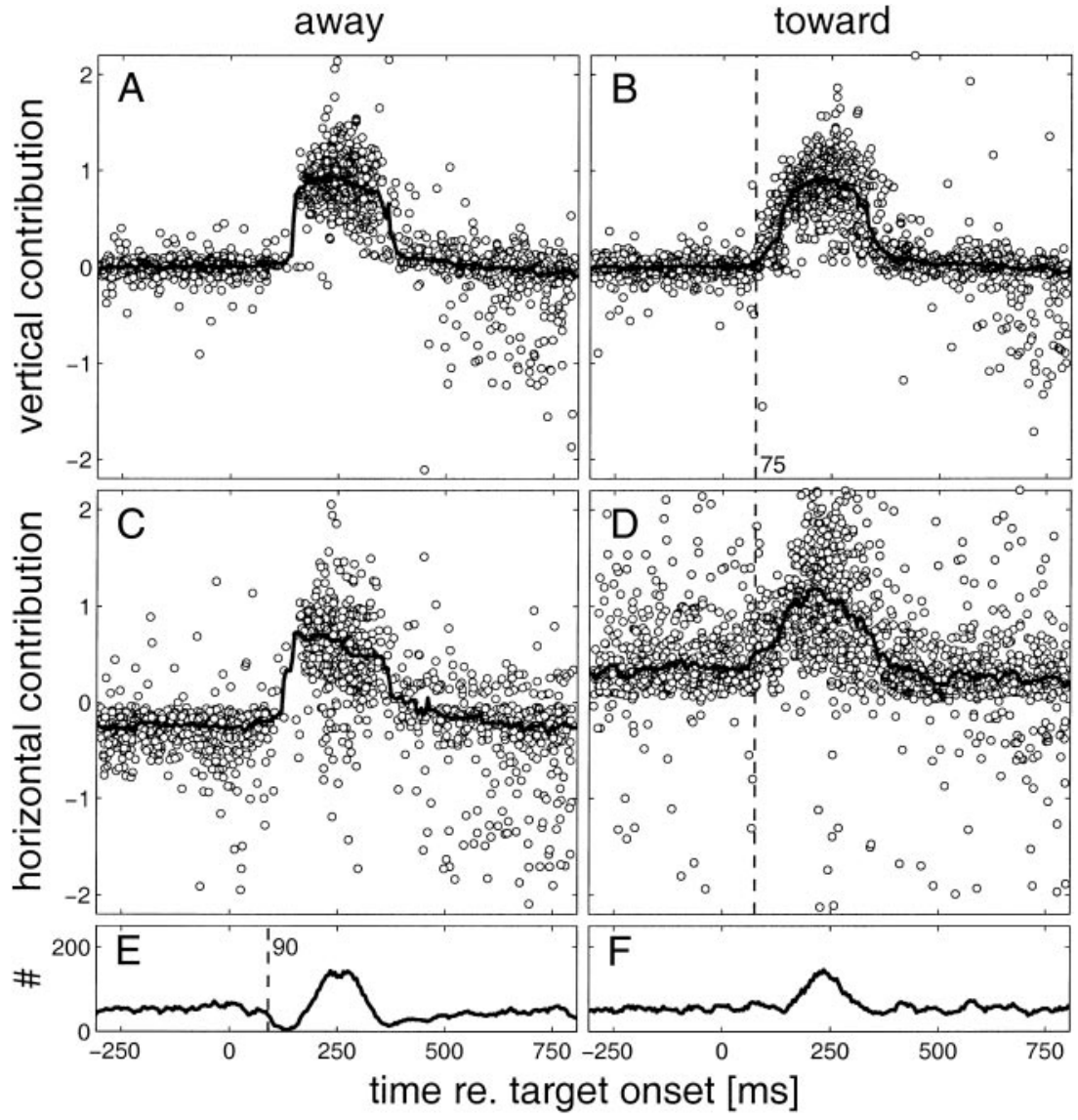

FIG. 7. Horizontal and vertical contributions in the prosaccade task. Same layout as in Fig. 6. Suppression of quick phases is only obvious in away trials. Note clear signs of early metric modification of both components in toward trials. Data pooled from all subjects except $P M$.

data still retain the early flash effects that we noticed already in the data from subject SP (see Fig. 6). In away trials, there was a clear rapid eye movement suppression starting $90 \mathrm{~ms}$ after target presentation. Moreover, from $75 \mathrm{~ms}$ after the flash, rapid eye movements in toward trials began showing a vertical target-directed component. A new feature, revealed by pooling, is that horizontal components of toward eye movements also show an early bias toward the flash. To illustrate that its onset was indistinguishable from the vertical-modification latency, we show that the dashed line in $B$ that marks vertical modification latency is also a good match to the horizontal data. So, the pooled data show that very early metric visual influences were present in both components at similar latencies. By contrast, there is no convincing evidence for early horizontal and vertical metrical modification in away trials (Fig. 7, $A$ and $C$ ).

\section{Effects of vestibular rotation in anti-saccade task}

QUICK-PHASE SUPPRESSION IN ANTI-SACCADE TASK. Our prosaccade results have shown that an early effect of the visual stimulus, occurring before the first goal-directed saccades, is to suppress reflexive rapid eye movements (quick phases) directed away from the target. This suppression makes sense from a functional point of view, but it might just be a rigid low-level mechanism, activated by the visual response to the flash. If, however, the suppression reflects a flexible strategy, one would expect it to adapt tactics when we change the task requirements by now asking the subject to make saccades away from the flash.

To investigate this issue, we performed a second series of experiments in the same subjects, using identical stimulus conditions to elicit anti-saccades rather than pro-saccades. Since the anti-saccade task was more demanding, the time interval between two consecutive flashes had to be prolonged so that we collected fewer responses in a single session than in the earlier pro-saccade experiments (see METHODS). Therefore we decided to focus the analysis on the pooled data from all five subjects.

We found that quick-phase suppression in the anti-saccade task was unmistakable in both types of trial (see Fig. 8, $E$ and $F)$. This result represents a marked departure from the prosaccade pattern, where suppression occurred only in away trials (see Figs. 6 and 7 and Table 1), with interesting implications concerning the nature of the quick-phase suppression. The fact that quick-phase suppression in toward trials did emerge on changing the oculomotor task seems to indicate the involvement of a flexible mechanism linked to task execution. On the other hand, the result that the suppression was still stronger and earlier in away trials ( $85 \mathrm{vs} .135 \mathrm{~ms}$ ), where it was least needed since these quick phases moved into the hemifield away from the flash, reveals a certain rigidity. Comparison with the pooled pro-saccade results (Fig. 7) suggests that suppression in away trials is virtually task-independent, an indication that this phenomenon is caused by a fast and inflexible process operating on a default basis.

METRICS IN THE ANTI-SACCADE TASK: LATENCY DEPENDENCE OF TASK PERFORMANCE. To visualize the changes in metrics of rapid eye movements as a function of latency, we constructed scatter plots depicting their normalized contribution values 


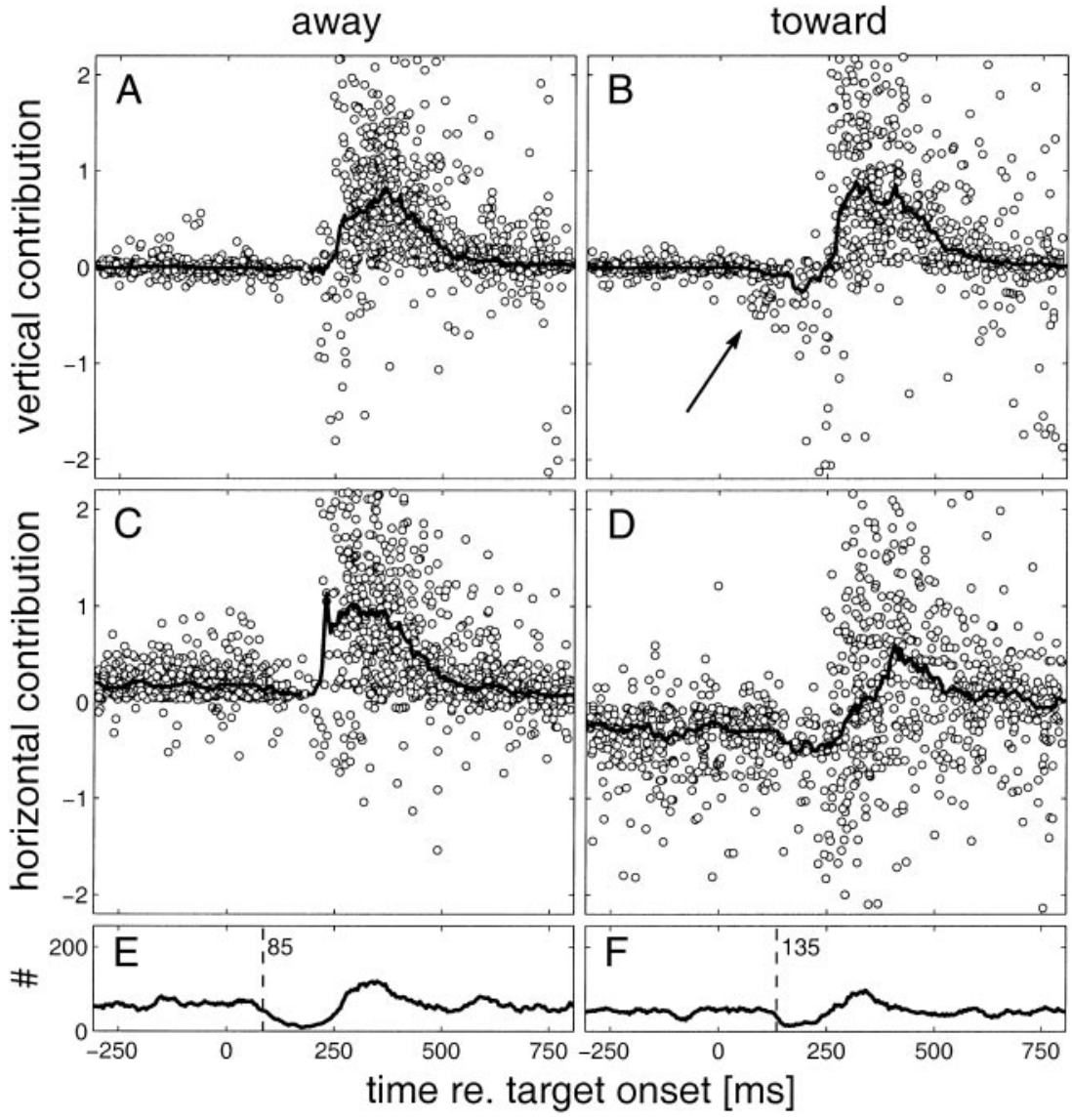

FIG. 8. Contributions in the anti-saccade task. Same layout as in Figs. 6 and 7. In the anti-saccade task, positive contributions indicate rapid eye movements toward the anti-position of the flash, whereas negative contributions represent eye movements in the direction of the flash. In the away condition, there was a rather clear separation between early pure quick phases and later, mostly correct, voluntary responses. In toward trials, error rates were much higher. At short latencies, a substantial number of eye movements had flash-directed vertical components (marked by arrow in $B$ ). The number of errors decreased with latency, 1st in the vertical component, slightly later in the horizontal component. Note that both in away and toward trials, there were clear signs of quick-phase suppression preceding the visually driven saccades. However, this was more prominent in the away condition. Data pooled from all subjects.

against time (see Fig. 8). As in the pro-saccade task, contributions were normalized with respect to the required refixation amplitude cued by the flash. Again, positive contributions mark eye movements into the direction of the required response. Negative values indicate incorrect eye movements, toward the flash. By the same definition, quick phases in the toward condition have negative contributions.

The simplest picture, with only mild signs of perturbation by vestibular rotation on anti-saccade task performance, was observed in away trials (left-hand column). The rapid eye movements in the time segment from before the flash until some 175 $\mathrm{ms}$ after its presentation, lacking any systematic metric effect of the flash, had the characteristics of pure quick phases. After an episode where relatively few rapid eye movements occurred (suppression period), we see a high density of apparently voluntary saccades. Most are in the required direction, but wrongly directed responses (negative contributions) are also in evidence, particularly in the short-latency range around 250 ms. So, in summary, away trials showed a rather neat distinction between early quick phases and later mostly correct voluntary saccades.

Toward trials, by contrast, showed many rapid eye movements in the direction of the flash, giving rise to a more complex picture involving several temporal stages. Again we see pure quick phases, unaffected by the flash, in the left-hand portion of the panels until approximately $60 \mathrm{~ms}$ after the flash. Next, we see the emergence of a first flash effect on metrics, marked by the arrow in Fig. $8 B$. In a limited time zone, starting here and overlapping partially with the suppression period, the vertical component develops a transient bias toward negative contributions. In other words, these components were directed toward the flash, violating the instruction to make anti-saccades. The corresponding horizontal contributions (Fig. 8D) show a tendency of having slightly larger flash-directed components than the pure quick phases earlier in the trial. Since these movements were absent in control and away trials, we suggest that these movements were vestibularly triggered but partially modified by the visual stimulus. We cannot exclude that some may have been voluntary. The problem is that, as far as the horizontal component is concerned, the vestibular drive and the flash in these toward trials would work in the same direction.

Ultimately, at longer latencies, the probability of correctly directed rapid eye movements increases also in toward trials. Interestingly, there is a clear indication in the data that this transition from flash-directed to correct anti-saccade responses is more abrupt and more complete in the vertical component. After $\sim 250 \mathrm{~ms}$, when most vertical responses are already in the anti-direction, there are still quite a number of wrongly directed horizontal components. For some reason, the horizontal channel has more difficulty to perform the task correctly, even at long latencies. To summarize, the first effect of the visual stimulus in toward trials was to attract quick phases toward the location of the flash by modifying their vertical component. The transition to correct responses, at longer latencies, occurred more abruptly and was more complete in the vertical than in the horizontal component. These indirect indications of some degree of independent behavior in the two components will now be substantiated further. 
CLASSIFICATION OF ANTISACCADE RESPONSES. In Fig. 9 we illustrate three toward trials with initially incorrect responses. Figure $9 A$ shows an initial response to the flash, in both components, which is subsequently corrected to bring gaze near the requested location (horizontal dashed lines) opposite to the flash. Note that the later corrective saccades are quite large, which helps explain why many saccades in Fig. 8 have large contributions. The short latency and the direction of the first eye movement are suggestive of a modified quick phase. The response in Fig. $9 B$ is more remarkable. Note that the horizontal component seems to start as a quick phase toward the flash, which was then modified in midflight (arrow) into the required direction. Strikingly, the vertical response was an almost flawless anti-response right from the start. Another striking example with clear signs of independent vertical and horizontal components is shown in Fig. 9C. Again the horizontal response is initially misguided and the vertical saccade is entirely correct. As a result, the eye initially ends up at a position far removed from both the flash location and the anti-target position (see panel on right-hand side).

To pursue the suggestion from these examples and the data in Fig. 8 that there was some degree of independence in the horizontal and vertical component of erroneous responses, we classified all horizontal and vertical refixation components independently. Four response types were distinguished:

1 ) wrong response, without correction later in trial,

2 ) wrong response, correction in saccade midflight,

3 ) wrong response, correction in later saccade(s),

4 ) correct anti-saccade response.

To exclude pure quick phases, which cannot be regarded as a response to the flash, all rapid eye movements with vertical components $<2^{\circ}$ and horizontal components into the direction of chair rotation were discarded. All trials were classified independently by both authors. In the classification procedure, automatic quick phase selections were checked and adjusted if necessary. In $11 \%$ of all cases, classification failed, either because there was no discernible response or because the response did not unambiguously fit one of the four categories. When an initial error was corrected in midflight or by later saccades (type 2 and 3 , respectively), a marker was set at the time of correction (see arrows in Fig. 9, $A$ and $B$ ).

Comparison of anti-saccade task performance in the three conditions (control, away, toward) revealed interesting differences (Table 2). In the stationary control condition, performance was good: from all classified trials, 94\% were correct anti-saccade responses in both the horizontal and the vertical component. During yaw rotation, performance deteriorated only slightly when quick phases were away from the flash. Generally, subjects were still quite able to shift gaze directly toward the anti-position, without first jumping toward the flash. Again, we found no major horizontal-vertical difference in performance. When quick phases were toward the visual target, however, performance worsened dramatically. Moreover, a major difference between the horizontal and vertical component emerged: in $45 \%$ of the refixations the horizontal component was initially directed toward the flash (classes 1, 2, and 3 combined), compared with only $19 \%$ in the vertical component. In $40 \%$ of the erroneous horizontal responses, errors were not corrected by later saccades (class 1). This marked difference between the error rates in the horizontal and vertical components clearly substantiates the earlier indications for a level of independence.
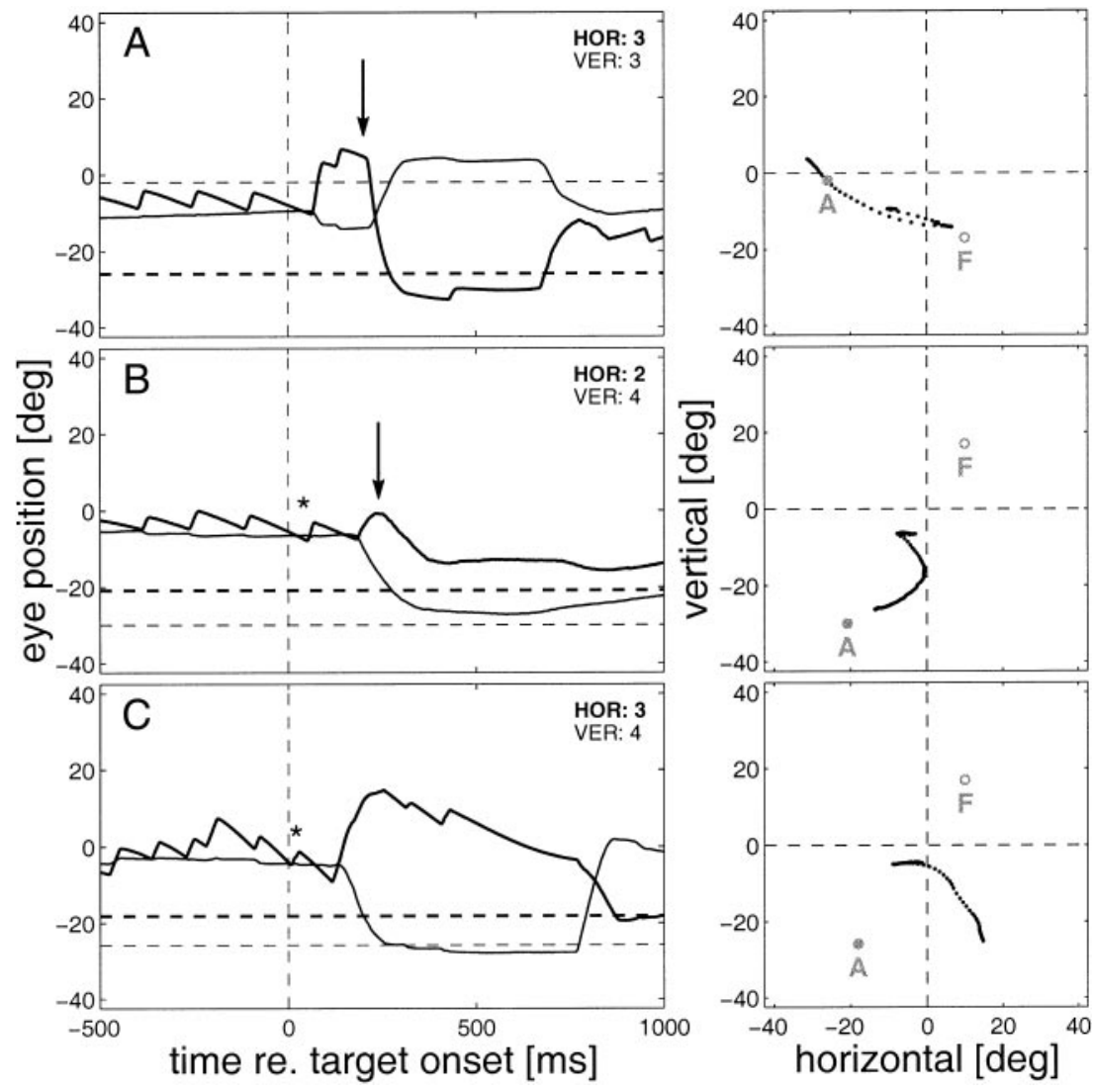

FIG. 9. Three examples of initially incorrect refixations in the anti-saccade task. The left-hand column shows horizontal (thick trace) and vertical (thin trace) eye position traces as a function of time. The vertical dashed line marks the time of the flash, the horizontal dashed lines denote the requested anti-response. The corresponding spatial trajectories are shown in the right-hand column, where $\mathrm{F}$ denotes flash location and A marks the anti-saccade target location. Note that the $\mathrm{A}$ is the mirrored position of the flash relative to the eye position at the moment of the flash, not relative to the straight ahead. Subjects often could not suppress eye movements that were directed toward the flash. Row A shows a response where both components were directed toward the flash and corrective saccades to the required anti-position occurred later. Row $B$ shows an incongruent response (vertical component correct; horizontal component incorrect) showing later correction in midflight. Row $C$ shows another incongruent response where the vertical component was directed toward the anti-target, whereas the horizontal component was toward the flash. Classifications (see text for details) are shown in the left-hand panels; arrows mark the time of correction. Pure quick phases immediately after the flash have been marked by an asterisk (*). Data from subjects $J G$ and $S P$. 
TABLE 2. Performance in the anti-saccade task

\begin{tabular}{|c|c|c|c|c|c|c|c|c|c|}
\hline Category & 1 & 2 or 3 & 4 & 1 & 2 or 3 & 4 & 1 & 2 or 3 & 4 \\
\hline Vertical & 2 & 4 & 94 & 5 & 9 & 87 & 4 & 15 & 81 \\
\hline
\end{tabular}

All refixations in the anti-saccade task were classified separately for each component, as explained in the text. Numbers denote occurrences as a percentage in class 1 , in combined classes 2 and 3 and in class 4 . In the stationary control condition, performance was quite good: $94 \%$ of the refixations showed the required anti response. Note that there was no difference in performance between the horizontal and vertical component. Only a slightly worsened performance was observed when subjects were rotated away from the flash. When rotated toward the flash, however, performance deteriorated dramatically, and a clear difference between the horizontal and vertical component emerged. Whereas $81 \%$ of the vertical refixation components was directed to the anti target, this was the case in only $55 \%$ of the horizontal components. Data pooled from all subjects.

DIFFERENT SPEED-ACCURACY RELATIONS FOR HORIZONTAL AND VERTICAL COMPONENTS. We noticed earlier (Fig. 8) that direction-error rates were higher in short-latency responses and that the speed-accuracy relation seemed different for horizontal and vertical. To explore these relationships further, Fig. 10 compares the latency distributions of the first visually influenced eye movements in correct refixations (open bars) and in trials that started with a movement toward the flash (filled bars). The top panels display the correct/incorrect latency distributions of the horizontal component, the bottom panels those of the vertical component.

Already at first sight, it is clear that controls and away trials show a similar pattern and that the toward trials stand out. Both the control and the away trials showed quite low direction-error rates in both components because error-prone short-latency responses were rare. As shown by the toward panels, performance deteriorated when the target was flashed into the direction of chair rotation. A major factor in the explanation is that, compared with the control and the away trials, a lot more early responses were made. Not too surprisingly, the vast majority of these premature responses was directed toward the flash, for both the horizontal and vertical component. However, the occurrence of premature responses is not the entire story. Note that the error-rates of horizontal and vertical components clearly show different time courses: whereas the proportion of errors in the vertical component became a minority at latencies beyond $\sim 250 \mathrm{~ms}$, incorrect horizontal responses were much more persistent and decayed very slowly from a much higher level.

In summary, there is a clear suggestion that rapid eye movements occurring early in toward trials tended to be incorrect, in both components. Incongruent responses, in which the vertical component was correct whereas the horizontal component was still directed toward the flash, emerged in an intermediate time zone.

To bring this better into focus, Fig. 11 shows refixation latency distributions of three classification subsets of the to-

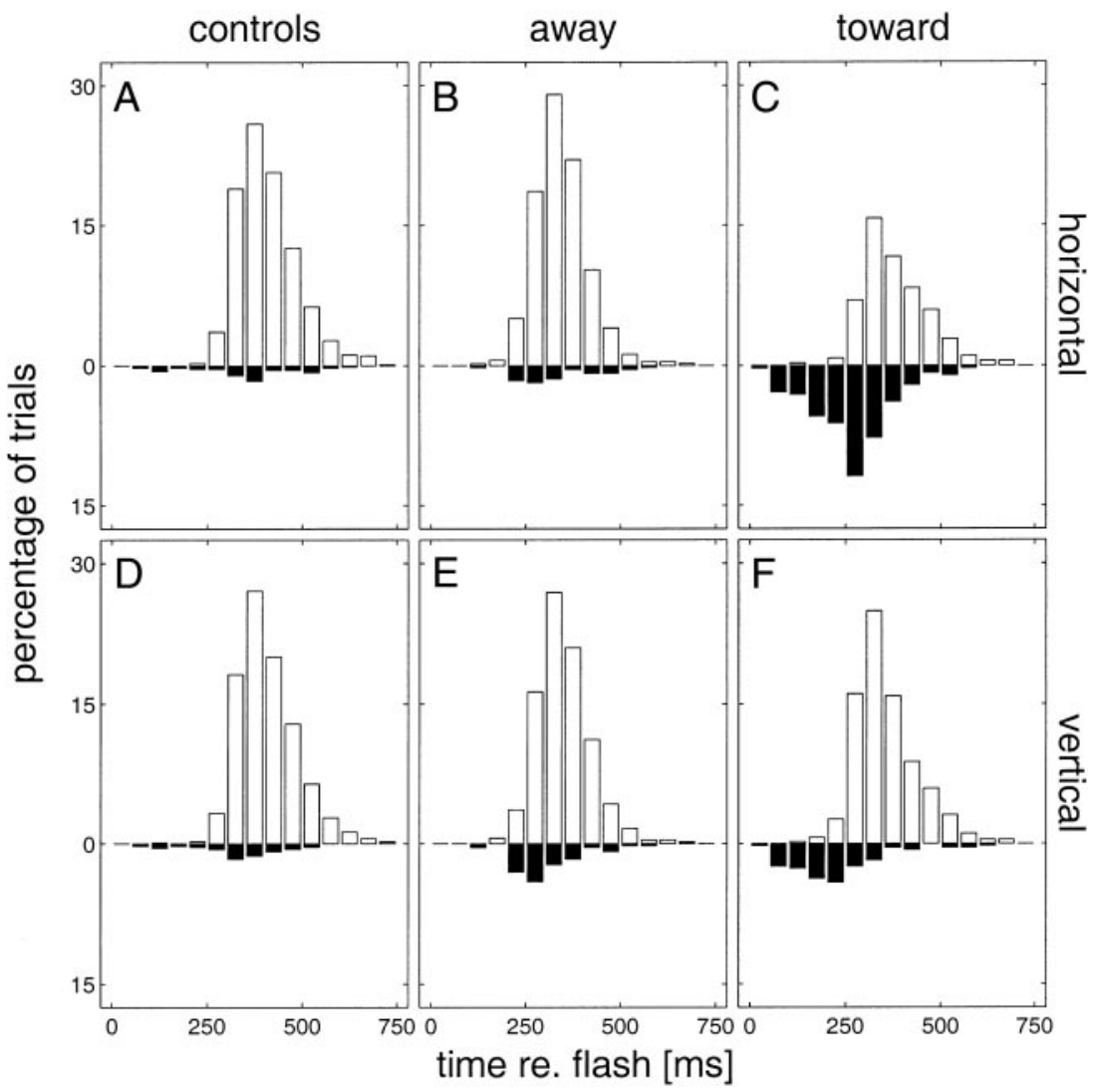

FIG. 10. Latency distributions of correct and initially incorrect responses in anti-saccade task. The top panels show the latency distributions of refixations with correct horizontal responses (that were classified 4, open bars) and of refixations whose horizontal component was initially directed toward the flash (that were classified either 1,2, or 3 , filled bars). The bottom panels show the corresponding distributions based on classifications of the vertical component. Note the high error rates in toward trials, especially in the horizontal component. Data pooled from all subjects. 


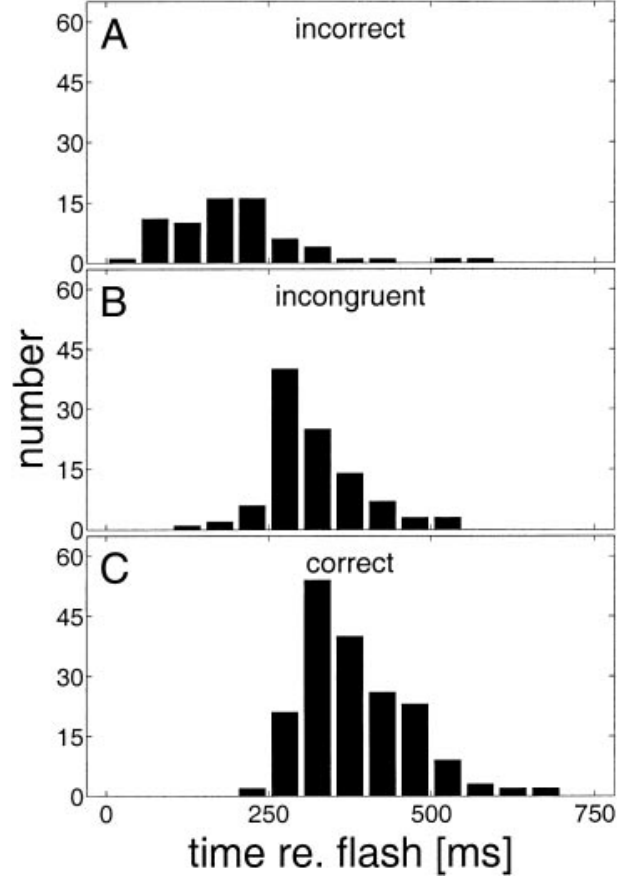

FIG. 11. Latency distributions in toward trials of anti-saccade task. To explore the transition from early incorrect responses to late correct responses, latency distributions of 2 subsets of incorrect trials $(A$ and $B)$ and of correct trials $(C)$ are shown. $A$ shows all trials in which both horizontal and vertical components were initially directed to the flash (classes 1,2 , or 3 ). At approximately $250 \mathrm{~ms}$, there is a clear shift to incongruent responses $(B)$ in which the vertical component was correct (class 4 ), but the horizontal component was directed to the flash. These responses comprised trials where the horizontal component was classified 1 or $3(n=79)$ and responses where the horizontal component was corrected in midflight (class $2, n=22$ ). These trials temporally overlap with correct anti refixations $(C)$. Pooled data from all subjects.

ward trials. Figure $11 \mathrm{~A}$ displays all trials in which both horizontal and vertical components of the first rapid eye movement were directed to the flash. The majority of these movements started early in the trial with a steep decrease in occurrence at $250 \mathrm{~ms}$ after the flash. From this time onward there was a prominent manifestation of incongruent trials in which the vertical component was correctly directed toward the antiposition right away, but the horizontal component initially was biased toward the flash and corrected later (Fig. 11B). Note that incongruent movements were made until very late in the trial. At a slightly later time, but showing clear overlap, refixations started in which both components were correct anti-saccade responses (Fig. 11C). Thus the impression gained from Fig. 10 is clearly confirmed: toward trials induced a substantial number of incongruent responses at a time when the anti-saccade signal was already available.

\section{I S C U S S I O N}

\section{Saccadic targeting errors during vestibular stimulation}

In all subjects, pro-saccade responses showed a clear deterioration of targeting accuracy during yaw rotation, especially in the horizontal component (see Figs. 2 and 3). These errors, however, did not simply reflect uncompensated vestibular eye movements. An analysis distinguishing the refixation displacements due to vestibular eye movements and saccades clearly showed that, on average, vestibular eye movements were taken into account, especially in trials where intervening quick phases were lacking (see Fig. 4). Our findings also exclude the possibility that subjects performed the task in a spatial frame of reference rather than head centered, as instructed. If subjects had made their saccadic responses to the remembered spatial location of the flash, one would expect undershoot responses in the toward trials and overshoot in away trials. If anything, the results show rather the opposite (see Fig. 4). In performing the refixation analysis, we based our definition of saccades on the vertical eye position channel, relying on the assumption that quick phases remained in the plane of rotation (see METHODS). As Fig. $1 C$ shows, the putative final vertical saccades could occur simultaneously with what looks like quick phase components in the horizontal channel. In these cases, involving a small corrective movement in the vertical channel, the distinction between a voluntary saccade and an involuntary quick phase could not be made with certainty. Since these final movements were small, their contribution to the total refixation was only minor. It is also important to note that only part of the data analysis depends on the refixation endpoint definition. The further analysis of pro- and anti-saccades (Figs. 6-11, Tables 1 and 2) does not depend on this definition.

In an earlier study (Van Beuzekom and Van Gisbergen 2002), we have investigated saccade-vestibular interactions in the monkey. The saccades were elicited by collicular electrical stimulation while the monkey was rotated. A difficulty in comparing the results is that electrical stimulation yielded single saccades, whereas the responses in the present study were often comprised of multiple saccades. While refixation responses in this paper showed clear signs of compensation for the intervening vestibular eye movements (see Fig. 4), this was rare in the monkey saccades elicited by electrical stimulation. A substantial number of sites actually showed robust metric changes that were anti-compensatory. An important difference between the two studies that may help to explain the difference in results is that in the monkey study the saccadic system was activated artificially by electrical stimulation at a late stage that bypassed cortical areas associated with saccade generation.

\section{Suppression of inappropriate rapid eye movements}

MECHANISMS FOR SUPPRESSING UNWANTED SACCADES. An important feature of the gaze control system is the ability to suppress unwanted saccades to nontarget stimuli. We simply do not want our eyes to shift to every change in our field of vision. This becomes essential in the anti-saccade paradigm, where attention needs to be shifted to the visual target to determine its location, without making a foveating saccade. So, to perform this task, the normal coupling between directing attention and shifting gaze needs to be suppressed, allowing time for the cue-related activity to subside and the anti-saccade signal to develop. Involvement of the frontal lobe in suppressing unwanted saccades has been suggested on the basis of data from neurological patients (Guitton et al. 1985). Possible neural correlates of such a process have been found at the level of the supplementary eye field (SEF) by Schlag-Rey et al. (1997) and in the frontal eye field (FEF) by Everling and Munoz (2000).

A somewhat similar situation is encountered in the countermanding task (Hanes and Carpenter 1999). Here, subjects are presented with a peripheral visual target when the fixation spot 
is switched off, but in a certain proportion of trials the fixation stimulus is lit again to signal that the response should be canceled. Neurophysiological experiments of this type in the monkey have shown that the saccade-related activity in the FEF is inhibited in successful abortion trials by increased fixation cell activity (Hanes et al. 1998).

How unwanted saccades can be prevented has also been studied at the level of the SC. Everling et al. (1999) recorded from fixation cells in the rostral zone and from buildup cells in the caudal area at the time when the monkey was awaiting the next anti-saccade trial. Comparison with activity levels in a pro-saccade trial showed that fixation cells were more active while buildup cells had decreased activity. This indicates that withholding saccades to the anti-saccade cue involves complementary changes at the level of the gaze holding mechanism (embodied by the fixation cells) and in the gaze-shifting system. To what extent these changes simply reflect the effect of incoming cortical signals is still unclear. Regardless, this work has shown that the $\mathrm{SC}$ is involved in implementing saccade suppression. Furthermore, by showing that these changes are already detectable even before the anti-cue is presented, the data have clearly established the precautionary nature of the suppression.

QUICK-PHASE SUPPRESSION. To our knowledge, the present study has been the first to provide a detailed description of saccade-target induced quick-phase suppression. It has been shown before that VOR suppression requires a visual stimulus, but these studies only concerned the slow phase of the VOR. Our quick-phase suppression results raise the question whether the cortical and collicular inhibition mechanisms discussed above may also be involved in the suppression of quick phases. A major difference between saccades and vestibular quick phases is that the latter cannot be suppressed by voluntary effort, without the help of a visual stimulus. Although there is evidence that quick phases are neurally represented in the colliculus (Schiller and Stryker 1972; Wurtz and Goldberg 1972), there is reason to doubt whether this signal plays a crucial role in generating quick phases. For example, quick phases of vestibular nystagmus can still be made after combined ablation of SC and FEF has abolished all visually evoked saccades (Schiller et al. 1980). Similarly, when the colliculus is inactivated by muscimol injection, quick phases can still be made (Hepp et al. 1993), but scanning saccades are suppressed. A further indication supporting the notion that quick-phase suppression is distinct from saccade suppression comes from a comparison of suppression latencies. Using the countermanding paradigm, Hanes and Carpenter (1999), found that the average stop-signal reaction time in humans was $137 \mathrm{~ms}$. This represents the time required for the visual stop signal to cancel a saccade that was being prepared. In our experiments, the time needed for a visual stimulus to stop quick phase generation was clearly much shorter, on average $100 \mathrm{~ms}$. While this difference is suggestive, caution is needed because the stop reaction times were obtained in different experiments and different subjects. In addition, the way how these latencies were determined was different as well. Since the probability of obtaining a visually guided saccade is itself a time-dependent process, Hanes and Carpenter had to base their assessment of stop-signal reaction time on a specific model. In our experiment, where the probability of getting a quick phase in the absence of a flash was simply constant, detection of the suppression latency was relatively straightforward (see Figs. 6-8).

If quick phases are generated downstream from the SC, as has been assumed in several modeling studies (Anastasio 1997; Galiana 1991), it becomes understandable why a strategy that prevents unwanted movements by modifying signals at the cortical or collicular level may fail to suppress quick phases. Taken together, there are good reasons to make a distinction between suppression of reflexive quick phases and of inadvertent pro-saccades in the anti-saccade task. As mentioned above, inadvertent pro-saccades can be prevented by precautionary mechanisms at the cortical and collicular level, but, apparently, such mechanisms have no grip on quick phases. The reason may be that quick phases are generated and suppressed in the brain stem.

POTENTIAL MECHANISM FOR QUICK-PHASE SUPPRESSION. Studies in the cat by Ohki et al. (1988) and by Kitama et al. (1995) on burster-driving neurons (BDNs) in and near the prepositus hypoglossus nucleus have revealed interesting functional properties of these cells that are thought to be an essential part of the quick-phase generator (for review, see Markham 1996). It should be noticed that BDNs, which have been extensively studied in the cat, have only been found in the vertical channel in the monkey, so far (Kaneko and Fukushima 1998). BDNs activated by vestibular rotation to the right contribute directly to the generation of the burst in excitatory burst neurons during vestibular quick phases in that direction. In addition, these cells have large visual receptive fields. For the BDNs activated during rightward quick phases and rightward rotation, a visual stimulus in the right hemifield will cause a short-latency excitatory response. Interestingly, a visual stimulus in the left hemifield, mimicking an away trial, causes a clear transient BDN suppression. It is possible to obtain the same effects by electrically stimulating the SC corresponding with each hemifield.

Although SC-BDN inhibition has not been discussed in this context before, the implications of this work for our prosaccade results seem rather clear. If BDNs generate quick phases, inhibiting them through the flash-evoked SC activity will cause quick-phase suppression after a short latency but only for quick phases directed away from the flash. Since BDN-mediated quick-phase suppression requires collicular activity, suppressing the SC by cortical intervention will be ineffective. This would explain why cortical intervention, which can prevent goal-directed saccades, fails to do so in quick phases. Notice also that this mechanism specifically targets quick phases, rather than all rapid eye movements equally.

The quick-phase suppression results in the anti-saccade task were more complex but may still fit in with our hypothesis. In these experiments, there was the same early strong suppression in away trials as we saw in pro-saccade experiments. We also observed a new phenomenon in the form of a weaker suppression, with a later onset, in toward trials. The simplest hypothesis to explain these findings is to assume that the transient SC response to the flash invokes the rapid and powerful taskindependent suppression mechanism found in the away trials of all experiments. We suggest that the later build up of antisaccade signals at the mirror site in the other colliculus may have caused the suppression in toward trials. 
Expression of visual-vestibular interaction in saccade metrics

PRO-SACCADE TASK. Toward trials in the pro-saccade task allowed us to study the latency-dependent transition from pure quick phases to voluntary goal-directed saccades. In these trials, where quick-phase suppression was not a complicating factor, we saw a first effect of the visual stimulus as early as 80 ms after its presentation. Since voluntary saccades had much longer latencies, we conclude that this phenomenon was due to visually modified quick phases. The flash-induced metric changes were most obvious in the vertical component (see Fig. 6) but became also discernible in the horizontal component after pooling (see Fig. 7). This phenomenon, that a rapid eye movement can combine visual and vestibular signals into a compromise response, invites comparison with earlier studies using visual double stimuli.

Becker and Jürgens (1979) investigated how compromise saccades can be elicited by a visual double-step stimulus. They showed that when the second target step occurred sufficiently early in the preparation period of the saccade, the eye landed on the final target position. If the shift was late in the preparation interval, close to saccade onset, the eye jumped to the initial target position. Interestingly, intermediate target steps yielded saccades that resembled a weighted vector average of the initial and final target positions and were therefore called "averaging saccades." Depending on the stimulus configuration, the effect of the second step became visible after a short latency, sometimes as short as $80 \mathrm{~ms}$. So, in view of our data, modification latency after a visual stimulus may be in the same range, irrespective of whether the initial rapid eye movement in preparation was a saccade or a quick phase.

ANTI-SACCADE TASK. The toward trials in the anti-saccade task were characterized by the large number of inadvertent rapid eye movements toward the flash early in the trial (see Fig. 8). To explain this phenomenon, we recall that in the toward trials, quick-phase suppression had a late onset and was incomplete. So, many eye movements were triggered when the flash response occurred in the SC and the corresponding anti-signal had not yet developed. When the pause cell gate was opened, the flash signal came to expression, yielding a biased quick phase. In away trials, hardly any quick phases were made in the period of flash-related activity in the SC, resulting in a low error rate.

In contrast to the pro-saccade results, where saccade metrics changed in synchrony for the horizontal and vertical component (see Fig. 7), anti-saccade toward trials showed clearly different temporal error profiles in the two components. In the horizontal component the error rate was much higher (see Table 2), and more persistent (see Fig. 10). Classification of all anti-saccade trials revealed an interesting time dependence in the error distributions (see Fig. 11). Not surprisingly, inadvertent pro-saccades were most frequently made shortly after the flash, whereas correct anti-saccades had longer latencies. Interestingly, in the intermediate time zone incongruent saccades were frequently made. In these responses, the vertical component was correctly directed toward the anti-target, whereas the horizontal component was aimed at the flash. In the next section, an attempt will be made to explain these bizarre movements.

\section{Possible neural basis of visual-vestibular averaging}

The pro-saccade results have shown clear evidence of visual-vestibular averaging. We found that vestibularly induced rapid eye movements became attracted toward the flash after about $80 \mathrm{~ms}$. Experiments with visual double stimuli at two different meridians (Chou et al. 1999; Ottes et al. 1984, 1985) have shown that averaging occurs only for a limited range of directional differences. When the two visual stimuli were presented on meridians that were at least $90^{\circ}$ apart, averaging responses gave way to a bistable response where either one or the other target attracted the saccade. The question is whether similar rules may apply to the present pro-saccade experiments, which involved a combination of a visual and a vestibular goal. If so, averaging would only be expected in toward trials where directional disparities were typically in the $30-60^{\circ}$ range. In away trials, opportunities for visual-vestibular averaging were unfavorable, at least seen from this perspective. Because of quick-phase suppression, quick phases were rare at the time when flash-evoked activity became available. When they did occur, the wide difference in direction between quick phases and the visual target would preclude averaging and yield either a pure pro-saccade or a pure quick phase. Indeed, we failed to see clear evidence for early modification in quick phases during away trials.

In the anti-saccade trials we saw again early modification of quick phases in toward trials. At short latencies, this phenomenon is not too surprising since quick phases and the flash occurred at closely spaced meridians (Fig. 12A). However, the rules specifying the conditions for saccade averaging in response to visual double targets cannot account for the phenomenon of incongruent responses (see Figs. 8 and 9C). To highlight this problem, Fig. $12 \mathrm{~B}$ shows the basic features of such a response, together with the signals that may be responsible. Note that the vertical response is correctly directed toward the required anti-location $(\mathrm{A})$ and that the horizontal response is incorrect, in the direction of the flash $(F)$ and of quick phases (Q). The fact that the vertical response is correct shows that the anti-saccade signal is already available. As shown in Fig. 11, a considerable proportion of rapid eye movements made in this time segment are entirely correct anti-saccades. Since the incongruent responses were only seen during vestibular rotation toward the flash, we suggest that occasional unsuppressed quick phases may have opened the pause-cell gate. Formally, the incongruent response can be seen as the result of an

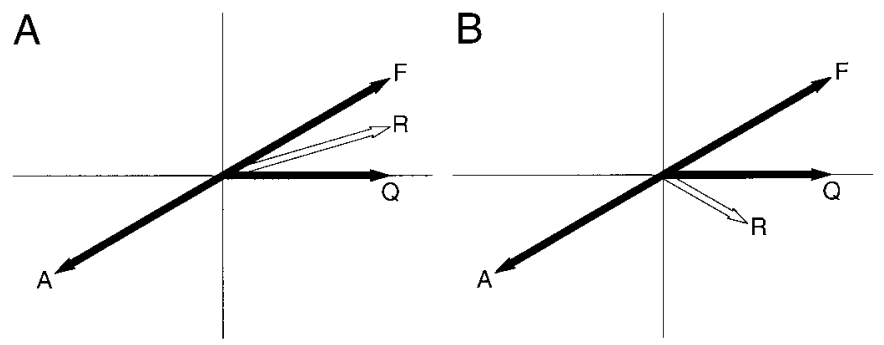

FIG. 12. Averaging in anti-saccade toward trials. The scheme illustrates 3 vectors competing for the control of rapid-eye movement responses. These correspond to the location of the flash $(\mathrm{F})$, the location of the anti-saccade target (A), and the goal for the quick phase (Q). A: when the flash signal predominates the anti-saccade signal, early in the trial, responses may reflect a compromise between F and Q. B: when the anti-saccade signal has developed, rapid eye movement initiated by the quick-phase system may give rise to a compromise response ( $\mathrm{R}$ ) directed in between $\mathrm{A}$ and $\mathrm{Q}$ (incongruent response). 
averaging computation involving anti-saccade signal $\mathrm{A}$ and quick-phase signal Q. What makes this averaging response remarkable is that it occurs despite a wide angular difference between vectors A and Q. From a functional point of view, the result is a bizarre response that is neither simply a pure quick phase or an inadvertent response to the flash, nor a correct anti-saccade. If averaging caused by visual-vestibular double stimulation is not following the same set of rules as for visual double stimulation, the underlying neural substrate is probably different in the two cases.

That visual double stimulation yields averaging for closely spaced stimuli but causes a selection process when the alternatives are farther apart has been discussed in terms of cooperation between adjacent areas within the SC motor map in conjunction with inhibitory interactions between more remote areas (Van Opstal and Van Gisbergen 1989). Long-range inhibitory interactions, which have now been shown in neurophysiological studies (Munoz and Istvan 1998) can explain why widely separated visual double stimuli do not yield averaging. Such a system can operate as a winner-take-all mechanism that weeds out either one or the other stimulus. Apparently, such all-or-nothing competitive mechanisms have not been operative in the anti-saccade experiments, otherwise incongruent responses would not have occurred. To explain their existence, the possibility has to be considered that saccade commands derived from the visual stimulus and quick-phase related activity did not compete because they occurred at different levels. This would be the case if the flash and antisaccade related activity affected the pulse generator via the SC input whereas quick-phase signals came in via the BDNs. Accordingly, the incongruent responses may represent addition of a collicular command for an anti-saccade and a brain stem command for a quick phase.

To visualize how this type of interaction may have worked, we consider the participating signals at various points in time after stimulus presentation. Figure 13 depicts how the time course of pro-saccade, anti-saccade, and quick-phase signals can account for the observed error-responses in toward trials in the anti-saccade task. It is assumed that interactions take place at the component level rather than at a vectorial coding stage. The flash, presented at time 0 , gives rise to short-latency transient activity. During the subsequent gradual decay of the flash-related activity, the anti-signal builds up to a steady level. It is assumed that quick phase activity, because of incomplete suppression, may be present until late in the trial. The bottom graphs show the eye movements predicted on the basis of the strengths of the three control signals, when the pause cell gate is opened and the control signals are tapped at various times after the flash. Early in the trial, only pure quick phases are made (response 1). During visual transient activity, inadvertent rapid eye movements attracted toward the flash can be elicited, triggered by the quick-phase generating mechanism (response 2 ). In the next time interval incongruent responses may occur (response 3). Since the anti-signal has overcome the flash signal, the former determines the vertical component. Its horizontal component, however, is dominated by the quick-phase activity, which, being horizontal, has no effect on the vertical component. The scheme indicates that incongruent responses may be corrected in midflight (response 4). Finally, correct fully voluntary responses are made when both flash and quickphase activity is suppressed (response 5).

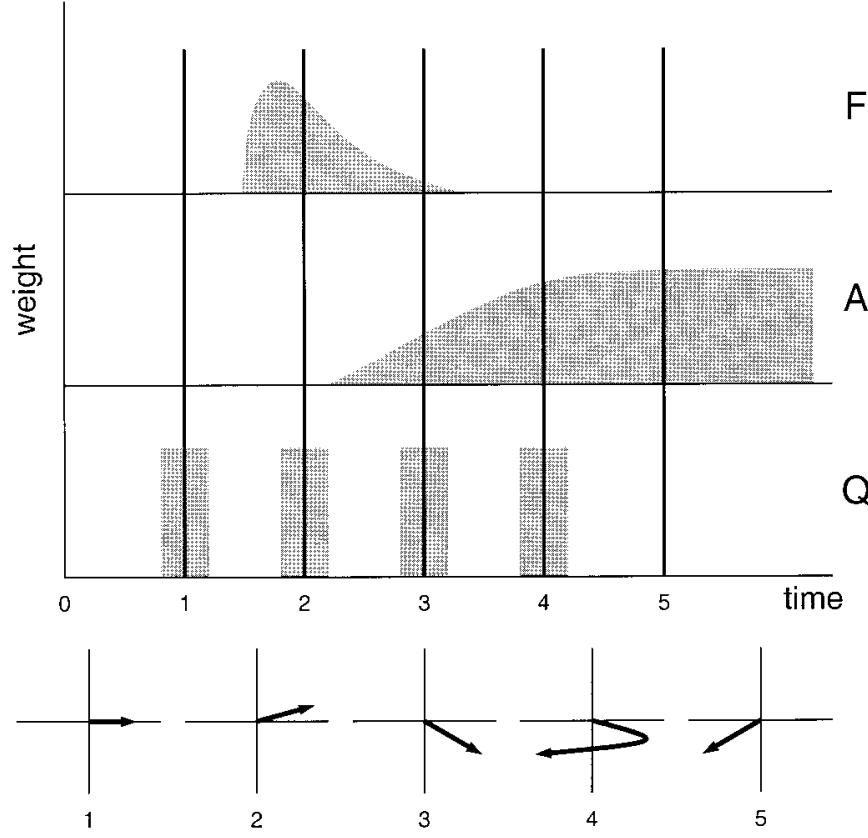

FIG. 13. Time course of visual and vestibular control signals in the antisaccade task and its relation to different response types. The top graphs show putative weights of the neural signals due to the flash $(\mathrm{F})$, the anti-saccade signal (A), and the vestibular quick-phase signal (Q) during a toward trial. The flash, presented at time 0 , results in a transient activity at a short latency. During the gradual decay of this transient activity, the anti-signal builds up to a steady level. Due to incomplete suppression, quick-phase activity may be present until late in toward trials. The bottom graphs show the eye movements predicted on the basis of the 3 control signals at 5 consecutive points in time (numbered 1 to 5). Early in the trial, pure quick phases are made (1). During visual transient activity, inadvertent pro-saccades can be elicited, triggered by the quick-phase generating mechanism (2). In the next stage, rapid eye movements induced by the quick-phase system are incorrect in their horizontal component but reflect the upcoming anti-saccade signal in their vertical component (3). The possibility of midflight correction of such incongruent responses has been indicated (4). From stage 3 onward, correct responses can be made when the quick-phase system happens to be inactive.

The authors thank J. Goossens for reading an earlier version of the manuscript and suggesting improvements. W. Corbeek and H. Van Brakel are acknowledged for essential technical support.

This work was supported by the Council for Earth and Life Sciences (ALW-NWO).

\section{REFERENCES}

ANASTASIO TJ. A burst-feedback model of fast-phase burst generation during nystagmus. Biol Cybern 76: 139-152, 1997.

BARR CC, SCHUltheIS LW, AND ROBINSON DA. Voluntary, non-visual control of the human vestibulo-ocular reflex. Acta Otolaryngol 81: 365-375, 1976.

BECKER W AND JÜRGENS R. An analysis of the saccadic system by means of double step stimuli. Vision Res 19: 967-983, 1979.

Chou IH, Sommer MA, AND Schiller PH. Express averaging saccades in monkeys. Vision Res 39: 4200-4216, 1999.

CollewiJn H, VAn der Mark F, AND Jansen TJ. Precise recording of human eye movements. Vision Res 15: 447-450, 1975.

EVERLING S, DORRIS MC, KLEIN RM, AND MUNOZ DP. Role of primate superior colliculus in preparation and execution of anti-saccades and pro-saccades. J Neurosci 19: 2740-2754, 1999.

EVERLING S AND MuNOZ DP. Neuronal correlates for preparatory set associated with pro-saccades and anti-saccades in the primate frontal eye field. $J$ Neurosci 20: 387-400, 2000.

GaLiana HL. A nystagmus strategy to linearize the vestibulo-ocular reflex. IEEE Trans Biomed Eng 38: 532-543, 1991.

GROH JM AND Sparks DL. Saccades to somatosensory targets. II. motor convergence in primate superior colliculus. J Neurophysiol 75: 428-438, 1996. 
Guitton D, Buchtel HA, And Douglas RM. Frontal lobe lesions in man cause difficulties in suppressing reflexive glances and in generating goaldirected saccades. Exp Brain Res 58: 455-472, 1985.

HANES DP AND CARPENTER RH. Countermanding saccades in humans. Vision Res 39: 2771-2791, 1999.

Hanes DP, Patterson WF II, AND Schall JD. Role of frontal eye fields in countermanding saccades: visual, movement, and fixation activity. $\mathrm{J} \mathrm{Neu}$ rophysiol 79: 817-834, 1998.

HepP K, Henn V, VILIS T, AND CoHen B. Brainstem regions related to saccade generation. In: The Neurobiology of Saccadic Eye Movements, edited by Wurtz RH and Goldberg ME. Amsterdam: Elsevier, 1989, p. 105-212.

Hepp K, Van Opstal AJ, Straumann D, Hess BJ, and Henn V. Monkey superior colliculus represents rapid eye movements in a two-dimensional motor map. J Neurophysiol 69: 965-979, 1993.

JAY MF AND SPARKS DL. Sensorimotor integration in the primate superior colliculus. I. Motor convergence. J Neurophysiol 57: 22-34, 1987.

KANEKo CR AND FuKUSHIMA K. Discharge characteristics of vestibular saccade neurons in alert monkeys. J Neurophysiol 79: 835-847, 1998.

KELLER EL. Participation of medial pontine reticular formation in eye movement generation in monkey. J Neurophysiol 37: 316-332, 1974.

Keller EL. The brainstem. In: Vision and Visual Dysfunction. Eye Movements, edited by Carpenter RHS. London: Macmillan, 1991, vol. 8, p. $200-223$.

Kitama T, Ohki Y, Shimazu H, Tanaka M, and Yoshida K. Site of interaction between saccade signals and vestibular signals induced by head rotation in the alert cat: functional properties and afferent organization of burster-driving neurons. J Neurophysiol 74: 273-289, 1995.

MARKHAM $\mathrm{CH}$. How does the brain generate horizontal nystagmus? In: Disorders of the Vestibular System, edited by Baloh RW and Halmagyi GM. Oxford, UK: Oxford Univ. Press, 1996, p. 48-61.

Melis BJ AND VAN Gisbergen JAM. Short-term adaptation of electrically induced saccades in monkey superior colliculus. J Neurophysiol 76: $1744-$ 1758, 1996.

Moschovakis AK, Scudder CA, And Highstein SM. The microscopic anatomy and physiology of the mammalian saccadic system. Prog Neurobiol 50: 133-254, 1996.

MunOz DP AND IsTVAN PJ. Lateral inhibitory interactions in the intermediate layers of the monkey superior colliculus. J Neurophysiol 79: 1193-1209, 1998.
OHKI Y, SHIMAZU H, AND SUZUKI I. Excitatory input to burst neurons from the labyrinth and its mediating pathway in the cat: location and functional characteristics of burster-driving neurons. Exp Brain Res 72: 457-472, 1988.

Ottes FP, VAn Gisbergen JA, And Eggermont JJ. Metrics of saccade responses to visual double stimuli: two different modes. Vision Res 24: 1169-1179, 1984.

OtTes FP, VAn Gisbergen JAM, And EgGermont JJ. Latency dependence of colour-based target vs nontarget discrimination by the saccadic system. Vision Res 25: 849-862, 1985.

RoBINSON DA. Eye movements evoked by collicular stimulation in the alert monkey. Vision Res 12: 1795-1808, 1972.

SCHILlER PH AND STRYKER M. Single-unit recording and stimulation in superior colliculus of the alert rhesus monkey. J Neurophysiol 35: 915-924, 1972.

SCHILlER PH, TRUE SD, AND CONWAy JL. Deficits in eye movements following frontal eye-field and superior colliculus ablations. J Neurophysiol 44: 11751189, 1980.

Schlag-Rey M, Amador N, Sanchez H, and Schlag J. Antisaccade performance predicted by neuronal activity in the supplementary eye field. Nature 390: $398-401,1997$.

SCUDDER CA, KANEKo CRS, AND FuCHS AF. The brainstem burst generator for saccadic eye movements. A modern synthesis. Exp Brain Res. 142: 439462, 2002.

SoKal R AND RohlF F. Biometry (2nd ed.). New York: Freeman, 1981.

SPARKS DL. Translation of sensory signals into commands for control of saccadic eye movements: role of primate superior colliculus. Physiol Rev 66: $118-171,1986$.

VAN BEUZEKOM AD AND VAN GISBERGEN JAM. Collicular microstimulation during passive rotation does not generate fixed gaze shifts. J Neurophysiol. 87: 2946-2963, 2002

VAN GisBergen JAM AND VAN BEUZEKOM A.D. Interference of nystagmus on prosaccade and antisaccade generation. Soc Neurosci Abstr 26-2: 1990, 2000.

VAN OPSTAL AJ AND VAN Gisbergen JAM. A nonlinear model for collicular spatial interactions underlying the metrical properties of electrically elicited saccades. Biol Cybern 60: 171-183, 1989.

WuRTZ RH AND GolDBERG ME. Activity of superior colliculus in behaving monkey. III. Cells discharging before eye movements. J Neurophysiol 35: 575-586, 1972. 